\title{
Guaranteed and robust a posteriori bounds for Laplace eigenvalues and eigenvectors: conforming approximations*
}

\author{
Eric Cancès ${ }^{\dagger} \quad$ Geneviève Dusson ${ }^{\ddagger \S} \quad$ Yvon Maday $^{\ddagger\|\|} \quad$ Benjamin Stamm** \\ Martin Vohralík ${ }^{\dagger \dagger}$
}

December 15, 2016

\begin{abstract}
This paper derives a posteriori error estimates for conforming numerical approximations of the Laplace eigenvalue problem with a homogeneous Dirichlet boundary condition. In particular, upper and lower bounds for an arbitrary simple eigenvalue are given. These bounds are guaranteed, fully computable, and converge with optimal speed to the given exact eigenvalue. They are valid without restrictions on the computational mesh or on the approximate eigenvector; we only need to assume that the approximate eigenvalue is separated from the surrounding smaller and larger exact ones, which can be checked in practice. Guaranteed, fully computable, optimally convergent, and polynomial-degree robust bounds on the energy error in the approximation of the associated eigenvector are derived as well, under the same hypotheses. Remarkably, there appears no unknown (solution-, regularity-, or polynomial-degree-dependent) constant in our theory, and no convexity/regularity assumption on the computational domain/exact eigenvector(s) is needed. Two improvements of the multiplicative constant appearing in our estimates are presented. First, it is reduced by a fixed factor under an explicit, a posteriori calculable condition on the mesh and on the approximate eigenvector-eigenvalue pair. Second, when an elliptic regularity assumption on the corresponding source problem is satisfied with known constants, the multiplicative factor can be brought to the optimal value of one. Inexact algebraic solvers are taken into account; the estimates are valid on each iteration and can serve for the design of adaptive stopping criteria. The application of our framework to conforming finite element approximations of arbitrary polynomial degree is provided, along with a numerical illustration on a set of test problems.
\end{abstract}

Key words: Laplace eigenvalue problem, a posteriori estimate, eigenvalue error, eigenvector error, guaranteed bound, conforming finite element method, inexact solver, stopping criteria

\section{Introduction}

Precise numerical approximation of eigenvalues and eigenvectors is crucial in countless applications. Thus, there has been a long-standing interest in answering the question: what is the size of the errors in computed

\footnotetext{
*This work was supported by the ANR project MANIF "Mathematical and numerical issues in first-principle molecular simulation". The last author has also received funding from the European Research Council (ERC) under the European Union's Horizon 2020 research and innovation program (grant agreement No 647134 GATIPOR).

†Université Paris Est, CERMICS, Ecole des Ponts and INRIA, 6 \& 8 Av. Pascal, 77455 Marne-la-Vallée, France (cances@cermics.enpc.fr).

¥Sorbonne Universités, UPMC Univ Paris 06, CNRS, UMR 7598, Laboratoire Jacques-Louis Lions, 4 place Jussieu 75005 , Paris, France (dusson@ann.jussieu.fr, maday@ann.jussieu.fr).

§Sorbonne Universités, UPMC Univ. Paris 06, Institut du Calcul et de la Simulation, 75005, Paris, France.

๑ Institut Universitaire de France, 75005, Paris, France.

"Division of Applied Mathematics, Brown University, 182 George St, Providence, RI 02912, USA.

** Center for Computational Engineering Science, RWTH Aachen University, Aachen, Germany and Computational Biomedicine, Institute for Advanced Simulation IAS-5 and Institute of Neuroscience and Medicine INM-9, Forschungszentrum Jülich, Germany (stamm@mathcces.rwth-aachen.de).

${ }^{\dagger \dagger}$ INRIA Paris, 2 rue Simone Iff, 75589 Paris, France (martin.vohralik@inria.fr).
} 
eigenvalues and eigenvectors? This question is usually tackled via a posteriori error estimates. For elliptic source problems such as the Laplace one, conclusive answers are today given by, in particular, the theory of equilibrated fluxes following Prager and Synge [49], see Destuynder and Métivet [19], Braess et al. [8], Ern and Vohralík [22], and the references therein. The structure of the Laplace eigenvalue problem appears rather richer in comparison with the elliptic source case.

Recently, though, there has been an important progress in obtaining guaranteed lower bounds for the eigenvalues, especially for the first one: Luo et al. [42], Hu et al. [32, 33], Carstensen and Gedicke [16], Yang et al. [63], or Liu [39] achieve so via the lowest-order nonconforming finite element method, Kuznetsov and Repin [37] and Šebestová and Vejchodský [54, 55] give numerical-method-independent estimates based on flux (functional) estimates, Liu and Oishi [41] elaborate fine a priori approximation estimates for lowestorder conforming finite elements, and, most recently, Xie et al. [62] also rely on fluxes. Earlier work comprises Kato [34], Forsythe [24], Weinberger [61], Bazley and Fox [4], Fox and Rheinboldt [25], Moler and Payne [45], Kuttler and Sigillito [35, 36], Still [57], Goerisch and He [27], Plum [48], Behnke et al. [5], and Armentano and Durán [1], see also the references therein. Sometimes, though, restrictions may apply. A condition on relative closeness to the (first) eigenvalue is necessary in [37, Remark 3.2], [54, condition (3.6)], and [55, condition (5.23)] (in these references, the bounds actually do not converge with the correct speed); solution of an auxiliary eigenvalue problem for nonconvex domains is requested [41]; potential overestimation on adaptively generated meshes may hamper the bounds of [41, 16, 39], relying on a priori estimates and employing the largest mesh element diameter; an auxiliary global flux problem needs to be solved in [62]; a saturation assumption may be necessary, see the discussion in [32].

The question of precision for both eigenvalues and eigenvectors has also been investigated previously. For conforming finite elements, relying on the a priori error estimates resumed in Babuška and Osborn [2] and Boffi [7], see also the references therein, a posteriori error estimates have been obtained by Verfürth [60], Maday and Patera [43], Larson [38], Heuveline and Rannacher [31], Durán et al. [20], Grubišić and Ovall [29], Rannacher et al. [50], and Šlín and Giani [56], see also the references therein. These estimates, though, systematically contain uncomputable terms, typically higher order on fine enough meshes.

Let $\Omega \subset \mathbb{R}^{d}, d=2,3$, be a polygonal/polyhedral domain with a Lipschitz boundary, and let $\lambda_{i}, u_{i}$ be the eigenvalues and associated eigenvectors of the Laplace operator $-\Delta$ on $\Omega$ with Dirichlet boundary conditions. The purpose of the present paper is to derive guaranteed and optimally convergent a posteriori bounds on both an arbitrary separated Laplace eigenvalue and the associated eigenvector for conforming (variational) methods. Nonconforming methods including nonconforming, discontinuous Galerkin, or mixed finite elements are treated in Cancès et al. [12]. We describe the setting in details in Section 2. Section 3 and Section 4 then contain a collection of equivalence inequalities between respectively the $i$-th eigenvalue error and the square of the $i$-th eigenvector energy error, the $i$-th eigenvector energy error and dual norm of the residual, and between the dual norm of the residual and its computable estimates. These results are valid under one key assumption: $\lambda_{i h}$ needs to be confined like $\lambda_{i-1}<\lambda_{i h}<\lambda_{i+1}$ (the left inequality of course only needs to hold when $i>1$ ), see (5.2) below. This can be guaranteed in many cases of practical interest by a domain inclusion argument $\Omega^{-} \subseteq \Omega \subseteq \Omega^{+}$with known smaller and larger eigenvalues $\lambda_{i-1} \leq \lambda_{i-1}\left(\Omega^{-}\right)$ and $\lambda_{i+1}\left(\Omega^{+}\right) \leq \lambda_{i+1}$ and by requesting $\overline{\bar{\lambda}}_{i-1}=: \lambda_{i-1}\left(\Omega^{-}\right)<\lambda_{i h}<\underline{\lambda}_{i+1}:=\lambda_{i+1}\left(\Omega^{+}\right)$. Numerical bounds $\bar{\lambda}_{i-1} \geq \lambda_{i-1}$ (typically available during the calculation) and $\underline{\lambda}_{i+1} \leq \lambda_{i+1}$ (obtained a on a coarse mesh by the approach of $[41,16,39])$ can also be used, see Remarks 5.3 and 5.4 below. We also suppose that the approximation spaces consist of appropriate piecewise polynomials. For improved versions of our bounds, we additionally need to check the smallness of the $L^{2}(\Omega)$-norm of the Riesz representation of the residual, see the a posteriori calculable conditions (5.6) and (5.9) below. These can be always satisfied by refining the computational mesh/increasing the polynomial degree of the approximate solution. Note that no condition of Galerkin orthogonality of the residual to the finite element hat functions needs to be satisfied: the entire analysis is presented in the context of inexact algebraic solvers. Our estimates are valid on each iteration subject to the above inclusion of $\lambda_{i h}$ and can be used for efficient adaptive stopping criteria of iterative eigenvalue solvers, as promoted in, e.g., Mehrmann and Miedlar [44] or Carstensen and Gedicke [16].

In Section 5, the results of Section 3-Section 4 are turned into actual a posteriori bounds. First, upper and lower bounds for the $i$-th eigenvalue are given in Theorems 5.1 and 5.2. For a finite element approximation with an exact algebraic solver for simplicity, we obtain

$$
\lambda_{i h}-\eta_{i}^{2} \leq \lambda_{i} \leq \lambda_{i h}-\tilde{\eta}_{i}^{2}
$$

with $\eta_{i}=m_{i h}\left\|\nabla u_{i h}+\boldsymbol{\sigma}_{i h, \text { dis }}\right\|$ and $\tilde{\eta}_{i}=\tilde{\eta}_{i}\left(r_{i h}\right)$ being fully computable quantities. Here $u_{i h}$ is the approxima- 
tion of the $i$-th exact eigenvector $u_{i},\|\cdot\|$ is the $L^{2}(\Omega)$-norm, $\sigma_{i h \text {, dis }}$ is an equilibrated flux reconstruction by mixed finite element local residual problems, and $r_{i h}$ is formed by conforming finite element local residual liftings. The associated eigenvector energy estimates are given next, with Theorem 5.6 revealing

$$
\left\|\nabla\left(u_{i}-u_{i h}\right)\right\| \leq \eta_{i}, \quad \eta_{i} \leq C_{i}\left\|\nabla\left(u_{i}-u_{i h}\right)\right\|,
$$

where $C_{i}$ is a constant that only depends on $\lambda_{1}, \bar{\lambda}_{i-1}, \lambda_{i h}, \underline{\lambda}_{i+1}$, on the space dimension $d$, and on some Poincaré-Friedrichs-type constant $C_{\text {cont,PF }}$ together with a discrete stability constant $C_{\text {st }}$, both only depending on the shape regularity of the mesh. In particular, $C_{i}$ is independent of the polynomial degree of $u_{i h}$, leading to the polynomial-degree robustness. Moreover, a computable bound on $C_{i}$ is given. The constant $C_{i}$, however, deteriorates for increasing eigenvalues. We distinguish three different cases. In Cases $\mathrm{A}$ and $\mathrm{B}$ of Theorems 5.1, 5.2, and 5.6, the multiplicative factor $m_{i h}$ of the estimator $\eta_{i}$ contains the factor $\max \left\{\left(\frac{\lambda_{i h}}{\bar{\lambda}_{i-1}}-1\right)^{-1},\left(1-\frac{\lambda_{i h}}{\underline{\lambda}_{i+1}}\right)^{-1}\right\}$ and similarly for $\tilde{\eta}_{i}$; Case B improves the overall size of $m_{i h}$ under the fine-enough-mesh condition (5.6). The results of these two cases hold without any assumption on the convexity of the computational domain $\Omega$ and on the regularity of the weak solutions. If, additionally, elliptic regularity of the corresponding source problem is known, the interpolation and stability constants are computable (typically when $d=2$ and $\Omega$ is convex), and the condition (5.9) holds, the factor $m_{i h}$ in front of the principal term $\left\|\nabla u_{i h}+\sigma_{i h \text {,dis }}\right\|$ has the optimal behavior $\sqrt{1+\mathcal{O}\left(h^{2}\right)}$, as summarized in Case $\mathrm{C}$ of Theorems 5.1, 5.2, and 5.6.

We show how to apply the above general results to conforming finite elements of arbitrary order in Section 6. Numerical experiments presented in Section 7 fully support the theoretical findings; in particular the necessary conditions hold from quite coarse meshes. We only treat here simple eigenvalues and associated eigenvectors; clustered and multiple eigenvalues will be dealt with in a forthcoming contribution. Finally, building on these results, guaranteed error bounds and fully adaptive strategies with dynamic stopping criteria may become possible for nonlinear eigenvalue problems; some of our first results in this direction are summarized in [11].

\section{Setting}

We denote by $H^{1}(\Omega)$ the Sobolev space of $L^{2}(\Omega)$ functions with weak gradients in $\left[L^{2}(\Omega)\right]^{d}$ and by $V:=$ $H_{0}^{1}(\Omega)$ its zero-trace subspace. Similarly, $\mathbf{H}(\operatorname{div}, \Omega)$ stands for the space of $\left[L^{2}(\Omega)\right]^{d}$ functions with weak divergences in $L^{2}(\Omega)$. The notations $\nabla$ and $\nabla$. are used respectively for the weak gradient and divergence. Moreover, for $\omega \subset \Omega,(\nabla u, \nabla v)_{\omega}$ stands for $\int_{\omega} \nabla u \cdot \nabla v \mathrm{~d} \mathbf{x}$ and $(u, v)_{\omega}$ for $\int_{\omega} u v \mathrm{~d} \mathbf{x}$; we also denote $\|\nabla v\|_{\omega}^{2}$ $:=\int_{\omega}|\nabla v|^{2} \mathrm{~d} \mathbf{x}$ and $\|v\|_{\omega}^{2}:=\int_{\omega} v^{2} \mathrm{~d} \mathbf{x}$ and drop the index whenever $\omega=\Omega$.

\subsection{The Laplace eigenvalue problem}

We consider here the problem: find eigenvector and eigenvalue pairs $\left(u_{k}, \lambda_{k}\right)$, with $u_{k}$ satisfying a homogeneous Dirichlet boundary condition over $\partial \Omega$ and subject to the constraint $\left\|u_{k}\right\|=1$, such that $-\Delta u_{k}=\lambda_{k} u_{k}$ in $\Omega$. In a weak form, $\left(u_{k}, \lambda_{k}\right) \in V \times \mathbb{R}^{+}$with $\left\|u_{k}\right\|=1$ and

$$
\left(\nabla u_{k}, \nabla v\right)=\lambda_{k}\left(u_{k}, v\right) \quad \forall v \in V .
$$

Actually, cf. Gilbarg and Trudinger [26], Babuška and Osborn [2], Boffi [7], or Strang and Fix [58], $u_{k}, k \geq 1$, form a countable orthonormal basis of $L^{2}(\Omega)$ consisting of vectors from $V$, whereas $0<\lambda_{1}<\lambda_{2} \leq \lambda_{3} \leq \ldots$ going to $+\infty$. The smallest eigenvalue $\lambda_{1}$ is positive and simple and the associated eigenvector $u_{k}$ to each simple $\lambda_{k}$ is unique up to the sign that we fix here by the condition $\left(u_{k}, \chi_{k}\right)>0$, where $\chi_{k} \in L^{2}(\Omega)$ is typically a characteristic function of $\Omega$ (for $k=1$ ) or of its subdomain (for $k>1$ ). Note that it follows from (2.1) and the scaling $\left\|u_{k}\right\|=1$ that $\left\|\nabla u_{k}\right\|^{2}=\lambda_{k}$.

Below, we shall often employ the Parseval identity, giving for any $v \in L^{2}(\Omega)$

$$
\|v\|^{2}=\sum_{k \geq 1}\left(v, u_{k}\right)^{2} .
$$


As $\left(u_{k} / \sqrt{\lambda_{k}}\right)_{k \geq 1}$ form an orthonormal basis of $V$, for which one in particular uses that $\left(\nabla u_{k}, \nabla u_{l}\right)=$ $\lambda_{k}\left(u_{k}, u_{l}\right)=0$ for $k \neq l$, for any $v \in V$, we also obtain

$$
\|\nabla v\|^{2}=\sum_{k \geq 1} \frac{\left(\nabla v, \nabla u_{k}\right)^{2}}{\lambda_{k}}=\sum_{k \geq 1} \lambda_{k}\left(v, u_{k}\right)^{2} .
$$

\subsection{Residual and its dual norm}

The derivation of a posteriori error estimates usually exploits the concept of the residual and of its dual norm. We will proceed in this way as well. Let $V^{\prime}$ stand for the dual of $V$.

Definition 2.1 (Residual and its dual norm). For any pair $\left(u_{i h}, \lambda_{i h}\right) \in V \times \mathbb{R}$, define the residual $\operatorname{Res}\left(u_{i h}, \lambda_{i h}\right) \in V^{\prime}$ by

$$
\left\langle\operatorname{Res}\left(u_{i h}, \lambda_{i h}\right), v\right\rangle_{V^{\prime}, V}:=\lambda_{i h}\left(u_{i h}, v\right)-\left(\nabla u_{i h}, \nabla v\right) \quad \forall v \in V .
$$

Its dual norm is then

$$
\left\|\operatorname{Res}\left(u_{i h}, \lambda_{i h}\right)\right\|_{-1}:=\sup _{v \in V,\|\nabla v\|=1}\left\langle\operatorname{Res}\left(u_{i h}, \lambda_{i h}\right), v\right\rangle_{V^{\prime}, V} .
$$

We will also often work with the Riesz representation of the residual $\boldsymbol{\varkappa}_{(i h)} \in V$,

$$
\begin{aligned}
\left(\nabla \varkappa_{i h}, \nabla v\right) & =\left\langle\operatorname{Res}\left(u_{i h}, \lambda_{i h}\right), v\right\rangle_{V^{\prime}, V} \quad \forall v \in V, \\
\left\|\nabla \xi_{(i h)}\right\| & =\left\|\operatorname{Res}\left(u_{i h}, \lambda_{i h}\right)\right\|_{-1} .
\end{aligned}
$$

\section{Generic equivalences}

In extension of some classical results, see $[26,2,7,58]$, we establish in this section generic equivalence results between the following three quantities: the $i$-th eigenvalue error $\left\|\nabla u_{i h}\right\|^{2}-\lambda_{i}$, which can potentially be negative, the square of the $i$-th eigenvector energy error $\left\|\nabla\left(u_{i}-u_{i h}\right)\right\|^{2}$, and the square of the dual norm of the residual $\left\|\operatorname{Res}\left(u_{i h}, \lambda_{i h}\right)\right\|_{-1}^{2}$. These equivalences may for the moment contain uncomputable terms like the eigenvalues $\lambda_{i-1}, \lambda_{i}, \lambda_{i+1}$ or the Riesz representation norm $\left\|\boldsymbol{\varkappa}_{i h)}\right\|$, but all such terms will be removed later. To proceed in an abstract way allowing for inexact algebraic solvers, we rather work with the eigenvalue error given by $\left\|\nabla u_{i h}\right\|^{2}-\lambda_{i}$ instead of $\lambda_{i h}-\lambda_{i}$; of course these coincide when the discrete Rayleigh quotient link $\left\|\nabla u_{i h}\right\|^{2}=\lambda_{i h}$ holds, typically upon solver convergence. A generalization to any self-adjoint operator with compact resolvent can be found in Cancès et al. [12].

Our first two lemmas are similar in parts to the developments in [35, 37, 54, 55], giving a computable bound on the $L^{2}(\Omega)$ error $\left\|u_{i}-u_{i h}\right\|$. Let $i \geq 1$ and define

$$
C_{i h}:=\min \left\{\left(1-\frac{\lambda_{i h}}{\lambda_{i-1}}\right)^{2},\left(1-\frac{\lambda_{i h}}{\lambda_{i+1}}\right)^{2}\right\} .
$$

The left term needs to be disregarded for $i=1$.

Lemma $3.1\left(L^{2}(\Omega)\right.$ bound via a quadratic residual inequality). Let $\left(u_{i h}, \lambda_{i h}\right) \in V \times \mathbb{R}^{+}$with $\left\|u_{i h}\right\|=1$ and $\left(u_{i}, u_{i h}\right) \geq 0$ be the $i$-th approximate eigenvector-eigenvalue pair, $i \geq 1$. Let $\lambda_{i}$ be simple and let $\lambda_{i-1}<\lambda_{i h}$ when $i>1$ and $\lambda_{i h}<\lambda_{i+1}$. Then

$$
\left\|u_{i}-u_{i h}\right\| \leq \alpha_{i h}:=\sqrt{2} C_{i h}^{-\frac{1}{2}}\left\|\boldsymbol{\varkappa}_{(i h)}\right\| .
$$

Proof. Characterizations (2.1), (2.4a), and (2.5a) give

$$
\left(\varkappa_{i h)}, u_{k}\right)=\frac{\left(\nabla u_{k}, \nabla \varkappa_{(i h)}\right)}{\lambda_{k}}=\frac{\left(\lambda_{i h}\left(u_{i h}, u_{k}\right)-\left(\nabla u_{i h}, \nabla u_{k}\right)\right)}{\lambda_{k}}=\left(\frac{\lambda_{i h}}{\lambda_{k}}-1\right)\left(u_{i h}, u_{k}\right) .
$$

Consequently, the Parseval equality (2.2) with $v=\boldsymbol{\varkappa}_{(i h)}$ yields

$$
\left\|\boldsymbol{\varkappa}_{(i h)}\right\|^{2}=\sum_{k \geq 1}\left(\boldsymbol{\varkappa}_{(i h)}, u_{k}\right)^{2}=\sum_{k \geq 1}\left(1-\frac{\lambda_{i h}}{\lambda_{k}}\right)^{2}\left(u_{i h}, u_{k}\right)^{2} .
$$


Observe that the function $x \in \mathbb{R}^{+} \mapsto\left(1-\frac{\lambda_{i h}}{x}\right)^{2}$ reaches its minimum at $x=\lambda_{i h}$ and is decreasing on $\left(0, \lambda_{i h}\right]$ and increasing on $\left[\lambda_{i h}, \infty\right)$. Thus the constant $C_{i h}$ in $(3.1)$ equals $\min _{k \geq 1, k \neq i}\left(1-\frac{\lambda_{i h}}{\lambda_{k}}\right)^{2}$. Further, employing the scalings $\left\|u_{i}\right\|=1$ and $\left\|u_{i h}\right\|=1$,

$$
\left(u_{i h}-u_{i}, u_{i}\right)=\left(u_{i h}, u_{i}\right)-\left\|u_{i}\right\|^{2}=\left(u_{i h}, u_{i}\right)-\frac{\left\|u_{i}\right\|^{2}}{2}-\frac{\left\|u_{i h}\right\|^{2}}{2}=-\frac{1}{2}\left\|u_{i}-u_{i h}\right\|^{2} .
$$

As $\left(u_{i}, u_{k}\right)=0$ for $k \geq 1, k \neq i$ from the orthogonality of $u_{k}$, elaborating (3.4) further while adding and subtracting $C_{i h}\left(u_{i h}-u_{i}, u_{i}\right)^{2}$ and using (3.1) and (3.5) gives

$$
\begin{aligned}
\left\|\boldsymbol{\xi}_{i h)}\right\|^{2} & =\left(\frac{\lambda_{i h}}{\lambda_{i}}-1\right)^{2}\left(u_{i h}, u_{i}\right)^{2}+\sum_{k \geq 1, k \neq i}\left(1-\frac{\lambda_{i h}}{\lambda_{k}}\right)^{2}\left(u_{i h}-u_{i}, u_{k}\right)^{2} \\
& \geq\left(\frac{\lambda_{i h}}{\lambda_{i}}-1\right)^{2}\left(u_{i h}, u_{i}\right)^{2}+C_{i h} \sum_{k \geq 1}\left(u_{i h}-u_{i}, u_{k}\right)^{2}-C_{i h}\left(u_{i h}-u_{i}, u_{i}\right)^{2} \\
& =\left(\frac{\lambda_{i h}}{\lambda_{i}}-1\right)^{2}\left(u_{i h}, u_{i}\right)^{2}+C_{i h}\left\|u_{i}-u_{i h}\right\|^{2}-\frac{C_{i h}}{4}\left\|u_{i}-u_{i h}\right\|^{4},
\end{aligned}
$$

where we have also employed (2.2) with $v=u_{i h}-u_{i}$. Dropping the first (nonnegative and presumably small) term on the right-hand side and denoting $e_{i h}:=\left\|u_{i}-u_{i h}\right\|^{2}$, we conclude the validity of the quadratic residual inequality in $e_{i h}$

$$
\frac{C_{i h}}{4} e_{i h}^{2}-C_{i h} e_{i h}+\left\|\boldsymbol{\imath}_{(i h)}\right\|^{2} \geq 0 .
$$

From the sign assumption $\left(u_{i}, u_{i h}\right) \geq 0$, employing $\left\|u_{i}\right\|=\left\|u_{i h}\right\|=1$,

$$
e_{i h}=\left\|u_{i}-u_{i h}\right\|^{2}=2-2\left(u_{i}, u_{i h}\right) \leq 2,
$$

so that $C_{i h} e_{i h} \leq 2\left\|\boldsymbol{\varkappa}_{i h}\right\|^{2}$, i.e., (3.2). Note that inspecting more closely the quadratic inequality (3.7), the improved bound $e_{i h} \leq 2-\sqrt{4-2 \alpha_{i h}^{2}}\left(\sqrt{2}\right.$-times better for $e_{i h}$ approaching zero) follows under condition $\left\|\boldsymbol{\xi}_{(i h)}\right\|^{2}<C_{i h}$ that we prefer to avoid.

In addition to (3.1), define also (disregarding again the left term for $i=1$ )

$$
\widetilde{C}_{i h}:=\min \left\{\lambda_{i-1}\left(1-\frac{\lambda_{i h}}{\lambda_{i-1}}\right)^{2}, \lambda_{i+1}\left(1-\frac{\lambda_{i h}}{\lambda_{i+1}}\right)^{2}\right\} .
$$

Lemma $3.2\left(L^{2}(\Omega)\right.$ bound with respect to $\left.\left\|\nabla \boldsymbol{\varkappa}_{i h)}\right\|\right)$. Under the assumptions of Lemma 3.1, there also holds

$$
\left\|u_{i}-u_{i h}\right\| \leq \alpha_{i h}:=\sqrt{2} \widetilde{C}_{i h}^{-\frac{1}{2}}\left\|\nabla \varkappa_{(i h)}\right\| .
$$

Proof. Developing (2.3) for $v=\varkappa_{i h}$ via (3.3) gives

$$
\left\|\nabla \boldsymbol{\varkappa}_{(i h)}\right\|^{2}=\sum_{k \geq 1} \lambda_{k}\left(\boldsymbol{\xi}_{(i h)}, u_{k}\right)^{2}=\sum_{k \geq 1} \lambda_{k}\left(1-\frac{\lambda_{i h}}{\lambda_{k}}\right)^{2}\left(u_{i h}, u_{k}\right)^{2} .
$$

Next, $\min _{k \geq 1, k \neq i} \lambda_{k}\left(1-\frac{\lambda_{i h}}{\lambda_{k}}\right)^{2}=\widetilde{C}_{i h}$. Thus, similarly to (3.6)-(3.7), with $e_{i h}:=\left\|u_{i}-u_{i h}\right\|^{2}, \frac{\widetilde{C}_{i h}}{4} e_{i h}^{2}-$ $\widetilde{C}_{i h} e_{i h}+\left\|\nabla \boldsymbol{r}_{(i h)}\right\|^{2} \geq 0$. One then concludes as in Lemma 3.1.

Recall the sign characterization $\left(u_{i}, \chi_{i}\right)>0$ with $\chi_{i} \in L^{2}(\Omega), i \geq 1$. The sign condition $\left(u_{i}, u_{i h}\right) \geq 0$ necessary in Lemmas 3.1 and 3.2 is typically always satisfied; the following lemma can be used for its rigorous verification:

Lemma 3.3 (Sign verification). Let $\left(u_{i h}, \lambda_{i h}\right) \in V \times \mathbb{R}^{+}$satisfy $\left\|u_{i h}\right\|=1,\left(u_{i h}, \chi_{i}\right)>0, \lambda_{i-1}<\lambda_{i h}$ when $i>1$ and $\lambda_{i h}<\lambda_{i+1}$, and $\alpha_{i h} \leq\left\|\chi_{i}\right\|^{-1}\left(u_{i h}, \chi_{i}\right)$ for $\alpha_{i h}$ given by (3.2) or (3.10). Then the sign condition $\left(u_{i}, u_{i h}\right) \geq 0$ is satisfied. 
Proof. Suppose $-\left(u_{i}, u_{i h}\right)>0$. Then the bounds of Lemmas 3.1 and 3.2 hold for $-u_{i h}$ in place of $u_{i h}$, i.e., $\left\|u_{i}+u_{i h}\right\| \leq \alpha_{i h}$. Consequently, a contradiction follows,

$$
\left(u_{i h}, \chi_{i}\right)=-\left(u_{i}, \chi_{i}\right)+\left(u_{i}+u_{i h}, \chi_{i}\right)<\left(u_{i}+u_{i h}, \chi_{i}\right) \leq\left\|u_{i}+u_{i h}\right\|\left\|\chi_{i}\right\| \leq\left(u_{i h}, \chi_{i}\right) .
$$

\section{$3.1 i$-th eigenvalue error equivalences}

We first show how to exploit the $L^{2}(\Omega)$ bound for equivalence between the eigenvalue error and the eigenvector error.

Theorem 3.4 (Eigenvalue bounds). Let $u_{i h} \in V$ with $\left\|u_{i h}\right\|=1, i \geq 1$, be arbitrary subject to $\left\|u_{i}-u_{i h}\right\| \leq$ $\alpha_{i h}$ for some $\alpha_{i h} \in \mathbb{R}^{+}$. Then

$$
\left\|\nabla\left(u_{i}-u_{i h}\right)\right\|^{2}-\lambda_{i} \alpha_{i h}^{2} \leq\left\|\nabla u_{i h}\right\|^{2}-\lambda_{i} \leq\left\|\nabla\left(u_{i}-u_{i h}\right)\right\|^{2} .
$$

Under the additional assumption $\alpha_{1 h}^{2} \leq 2$, there also holds, for the first eigenpair,

$$
\frac{1}{2}\left(1-\frac{\lambda_{1}}{\lambda_{2}}\right)\left(1-\frac{\alpha_{1 h}^{2}}{4}\right)\left\|\nabla\left(u_{1}-u_{1 h}\right)\right\|^{2} \leq\left\|\nabla u_{1 h}\right\|^{2}-\lambda_{1} .
$$

Proof. Using the weak solution characterization (2.1) and (3.5),

$$
\begin{aligned}
\left\|\nabla u_{i h}\right\|^{2}-\lambda_{i} & =\left\|\nabla\left(u_{i h}-u_{i}\right)\right\|^{2}+2\left(\nabla\left(u_{i h}-u_{i}\right), \nabla u_{i}\right) \\
& =\left\|\nabla\left(u_{i h}-u_{i}\right)\right\|^{2}+2 \lambda_{i}\left(u_{i}, u_{i h}-u_{i}\right) \\
& =\left\|\nabla\left(u_{i h}-u_{i}\right)\right\|^{2}-\lambda_{i}\left\|u_{i}-u_{i h}\right\|^{2} .
\end{aligned}
$$

Dropping the (nonpositive and presumably small) last term, the upper bound in (3.12) follows; estimating it using $\left\|u_{i}-u_{i h}\right\| \leq \alpha_{i h}$, we arrive at the lower bound in (3.12). First,

The bound (3.13) only seems to hold for the first eigenpair. To prove it, we use (2.2)-(2.3) for $v=u_{1}-u_{1 h}$.

$$
\left\|\nabla\left(u_{1}-u_{1 h}\right)\right\|^{2}-\lambda_{1}\left\|u_{1}-u_{1 h}\right\|^{2}=\sum_{k \geq 1}\left(\lambda_{k}-\lambda_{1}\right)\left(u_{1}-u_{1 h}, u_{k}\right)^{2}=\sum_{k \geq 2}\left(\lambda_{k}-\lambda_{1}\right)\left(u_{1}-u_{1 h}, u_{k}\right)^{2} .
$$

Using $\lambda_{k} \geq \lambda_{2}$ for $k \geq 2, \lambda_{2}>\lambda_{1}$, (3.5) for $i=1$, and the Cauchy-Schwarz inequality,

$$
\begin{aligned}
\left\|\nabla\left(u_{1}-u_{1 h}\right)\right\|^{2}-\lambda_{1}\left\|u_{1}-u_{1 h}\right\|^{2} & \geq\left(\lambda_{2}-\lambda_{1}\right) \sum_{k \geq 1}\left(u_{1}-u_{1 h}, u_{k}\right)^{2}-\left(\lambda_{2}-\lambda_{1}\right)\left(u_{1}-u_{1 h}, u_{1}\right)^{2} \\
& =\left(\lambda_{2}-\lambda_{1}\right)\left\|u_{1}-u_{1 h}\right\|^{2}-\frac{\lambda_{2}-\lambda_{1}}{4}\left\|u_{1}-u_{1 h}\right\|^{4} .
\end{aligned}
$$

Using $\left\|u_{1}-u_{1 h}\right\| \leq \alpha_{1 h}$ and reemploying (2.2) for $v=u_{1}-u_{1 h}$, we arrive at, second,

$$
\begin{aligned}
\left\|\nabla\left(u_{1}-u_{1 h}\right)\right\|^{2}-\lambda_{1}\left\|u_{1}-u_{1 h}\right\|^{2} & \geq\left(\lambda_{2}-\lambda_{1}\right)\left\|u_{1}-u_{1 h}\right\|^{2}-\alpha_{1 h}^{2} \frac{\lambda_{2}-\lambda_{1}}{4}\left\|u_{1}-u_{1 h}\right\|^{2} \\
& =\sum_{k \geq 1}\left(\lambda_{2}-\lambda_{1}\right)\left(1-\frac{\alpha_{1 h}^{2}}{4}\right)\left(u_{1}-u_{1 h}, u_{k}\right)^{2} .
\end{aligned}
$$

Summing this with (3.15) with weights $\frac{1}{2}$ yields

$$
\left\|\nabla\left(u_{1}-u_{1 h}\right)\right\|^{2}-\lambda_{1}\left\|u_{1}-u_{1 h}\right\|^{2} \geq \sum_{k \geq 1}\left\{\frac{\lambda_{k}-\lambda_{1}}{2}+\frac{\lambda_{2}-\lambda_{1}}{2}\left(1-\frac{\alpha_{1 h}^{2}}{4}\right)\right\}\left(u_{1}-u_{1 h}, u_{k}\right)^{2} .
$$

Now notice that, using (2.3) for $v=u_{1}-u_{1 h}$,

$$
\frac{1}{2}\left(1-\frac{\lambda_{1}}{\lambda_{2}}\right)\left(1-\frac{\alpha_{1 h}^{2}}{4}\right)\left\|\nabla\left(u_{1}-u_{1 h}\right)\right\|^{2}=\sum_{k \geq 1} \frac{\lambda_{k}}{2}\left(1-\frac{\lambda_{1}}{\lambda_{2}}\right)\left(1-\frac{\alpha_{1 h}^{2}}{4}\right)\left(u_{1}-u_{1 h}, u_{k}\right)^{2} .
$$


A simple calculation (note $\frac{1}{2} \leq\left(1-\frac{\alpha_{1 h}^{2}}{4}\right) \leq 1$ ) shows that

$$
\frac{\lambda_{k}-\lambda_{1}}{2}+\frac{\lambda_{2}-\lambda_{1}}{2}\left(1-\frac{\alpha_{1 h}^{2}}{4}\right) \geq \frac{\lambda_{k}}{2}\left(1-\frac{\lambda_{1}}{\lambda_{2}}\right)\left(1-\frac{\alpha_{1 h}^{2}}{4}\right) \quad k \geq 1 .
$$

Thus

$$
\left\|\nabla\left(u_{1}-u_{1 h}\right)\right\|^{2}-\lambda_{1}\left\|u_{1}-u_{1 h}\right\|^{2} \geq \frac{1}{2}\left(1-\frac{\lambda_{1}}{\lambda_{2}}\right)\left(1-\frac{\alpha_{1 h}^{2}}{4}\right)\left\|\nabla\left(u_{1}-u_{1 h}\right)\right\|^{2},
$$

and (3.13) follows using (3.14).

\section{$3.2 \quad i$-th eigenvector error equivalences}

We next investigate the equivalence between the eigenvector error $\left\|\nabla\left(u_{i}-u_{i h}\right)\right\|$ and the dual norm of the residual $\left\|\operatorname{Res}\left(u_{i h}, \lambda_{i h}\right)\right\|_{-1}$. Recall the definition (3.1) and also set

$$
\bar{C}_{i h}:=1 \text { if } i=1, \quad \bar{C}_{i h}:=\max \left\{\left(\frac{\lambda_{i h}}{\lambda_{1}}-1\right)^{2}, 1\right\} \text { if } i>1 .
$$

Furthermore, let

$$
\gamma_{i h}:= \begin{cases}\left\|\nabla\left(u_{i}-u_{i h}\right)\right\|^{2} & \text { if } \lambda_{i} \leq\left\|\nabla u_{i h}\right\|^{2} \text { is known to hold, } \\ \max \left\{\left\|\nabla\left(u_{i}-u_{i h}\right)\right\|^{2}, \lambda_{i} \alpha_{i h}^{2}\right\} & \text { otherwise; }\end{cases}
$$

we refer to Remark 5.4 below for the discussion when $\lambda_{i} \leq\left\|\nabla u_{i h}\right\|^{2}$.

Theorem 3.5 (Eigenvector bounds). Let $\left(u_{i h}, \lambda_{i h}\right) \in V \times \mathbb{R}^{+}$with $\left\|u_{i h}\right\|=1, i \geq 1$, be arbitrary subject to $\left\|u_{i}-u_{i h}\right\| \leq \alpha_{i h}$ for some $\alpha_{i h} \in \mathbb{R}^{+}$. Then

$$
\begin{aligned}
\left\|\nabla\left(u_{i}-u_{i h}\right)\right\|^{2} & \leq\left\|\operatorname{Res}\left(u_{i h}, \lambda_{i h}\right)\right\|_{-1}^{2}+\left(\lambda_{i h}+\lambda_{i}\right) \alpha_{i h}^{2}, \\
\left\|\operatorname{Res}\left(u_{i h}, \lambda_{i h}\right)\right\|_{-1}^{2} & \leq \frac{\left(\left|\lambda_{i h}-\left\|\nabla u_{i h}\right\|^{2}\right|+\gamma_{i h}\right)^{2}}{\lambda_{i}}+\bar{C}_{i h}\left\|\nabla\left(u_{i}-u_{i h}\right)\right\|^{2} .
\end{aligned}
$$

Let $\lambda_{i-1}<\lambda_{\text {ih }}$ when $i>1, \lambda_{i h}<\lambda_{i+1}$, and $\alpha_{i h}^{2} \leq 2 \frac{\lambda_{1}}{\lambda_{i}}$. Then there also holds

$$
\left\|\nabla\left(u_{i}-u_{i h}\right)\right\|^{2} \leq C_{i h}^{-1}\left(1-\frac{\lambda_{i}}{\lambda_{1}} \frac{\alpha_{i h}^{2}}{4}\right)^{-1}\left\|\operatorname{Res}\left(u_{i h}, \lambda_{i h}\right)\right\|_{-1}^{2} .
$$

Proof. Starting from (3.11), adding and subtracting $C_{i h} \lambda_{i}\left(u_{i h}-u_{i}, u_{i}\right)^{2}$, using $\left(u_{i}, u_{k}\right)=0$ for $k \geq 1, k \neq i$, (3.5), and the Cauchy-Schwarz inequality, we observe

$$
\begin{aligned}
\left\|\nabla \xi_{i h)}\right\|^{2} & \geq \lambda_{i}\left(\frac{\lambda_{i h}}{\lambda_{i}}-1\right)^{2}\left(u_{i h}, u_{i}\right)^{2}+C_{i h} \sum_{k \geq 1} \lambda_{k}\left(u_{i h}-u_{i}, u_{k}\right)^{2}-C_{i h} \lambda_{i}\left(u_{i h}-u_{i}, u_{i}\right)^{2} \\
& =\lambda_{i}\left(\frac{\lambda_{i h}}{\lambda_{i}}-1\right)^{2}\left(u_{i h}, u_{i}\right)^{2}+C_{i h}\left\|\nabla\left(u_{i}-u_{i h}\right)\right\|^{2}-\frac{C_{i h}}{4} \lambda_{i}\left\|u_{i}-u_{i h}\right\|^{4} \\
& \geq C_{i h}\left\|\nabla\left(u_{i}-u_{i h}\right)\right\|^{2}-\frac{C_{i h}}{4} \lambda_{i}\left\|u_{i}-u_{i h}\right\|^{4} .
\end{aligned}
$$

Using the Poincaré-Friedrichs inequality $\left\|u_{i}-u_{i h}\right\|^{2} \leq \frac{1}{\lambda_{1}}\left\|\nabla\left(u_{i}-u_{i h}\right)\right\|^{2}$,

$$
\left\|\nabla \varkappa_{i h)}\right\|^{2} \geq C_{i h}\left\|\nabla\left(u_{i}-u_{i h}\right)\right\|^{2}-\frac{C_{i h}}{4} \frac{\lambda_{i}}{\lambda_{1}}\left\|\nabla\left(u_{i}-u_{i h}\right)\right\|^{2} \alpha_{i h}^{2},
$$

where we have also employed $\left\|u_{i}-u_{i h}\right\| \leq \alpha_{i h}$. Thus (3.19) follows via (2.5b). 
The proof of Lemma 3.1 gives $\sup _{k \geq 1, k \neq i}\left(1-\frac{\lambda_{i h}}{\lambda_{k}}\right)^{2}=\bar{C}_{i h}$, recalling (3.16). Thus, (3.11) together with the Cauchy-Schwarz inequality and $\left\|u_{i}\right\|=\left\|u_{i h}\right\|=1$ give

$$
\begin{aligned}
\left\|\nabla \varkappa_{i h)}\right\|^{2} & \leq \lambda_{i}\left(\frac{\lambda_{i h}}{\lambda_{i}}-1\right)^{2}+\bar{C}_{i h} \sum_{k \geq 1, k \neq i} \lambda_{k}\left(u_{i h}-u_{i}, u_{k}\right)^{2} \\
& \leq \frac{\left(\lambda_{i h}-\lambda_{i}\right)^{2}}{\lambda_{i}}+\bar{C}_{i h}\left\|\nabla\left(u_{i}-u_{i h}\right)\right\|^{2} .
\end{aligned}
$$

Using the inequalities (3.12) and the definition (3.17) of $\gamma_{i h}$,

$$
\left|\lambda_{i h}-\lambda_{i}\right| \leq\left|\lambda_{i h}-\left\|\nabla u_{i h}\right\|^{2}\right|+\left|\left\|\nabla u_{i h}\right\|^{2}-\lambda_{i}\right| \leq\left|\lambda_{i h}-\left\|\nabla u_{i h}\right\|^{2}\right|+\gamma_{i h},
$$

so that $(3.18 \mathrm{~b})$ is proven.

Finally, (3.18a) can be seen as in, e.g., Carstensen and Gedicke [15, Lemma 3.1] combined with $\| u_{i}-$ $u_{i h} \| \leq \alpha_{i h}[-]$.

\section{Dual norm of the residual equivalences}

We now estimate the dual residual norm $\left\|\operatorname{Res}\left(u_{i h}, \lambda_{i h}\right)\right\|_{-1}$, for $u_{i h} \in V$ a piecewise polynomial of degree $p \geq 1$ and $\lambda_{i h} \in \mathbb{R}$. For the upper bound, following [49, 19, 8, 22] and [21, 47, 46] for inexact solvers, see also the references therein, we introduce an equilibrated flux reconstruction. This is a vector field $\boldsymbol{\sigma}_{i h}$ constructed from the local residual of $\left(u_{i h}, \lambda_{i h}\right)$ by solving patchwise mixed finite element problems such that

$$
\begin{aligned}
\boldsymbol{\sigma}_{i h} & \in \mathbf{V}_{h} \subset \mathbf{H}(\operatorname{div}, \Omega), \\
\nabla \cdot \boldsymbol{\sigma}_{i h} & =\lambda_{i h} u_{i h}-\rho_{i h}, \quad \lambda_{1}^{-\frac{1}{2}}\left\|\rho_{i h}\right\| \text { sufficiently small. }
\end{aligned}
$$

Inversely, local conforming residual liftings following [3, Section 5.1], [51, Section 4.1.1], [22, Section 3.3] will allow us to construct $r_{i h} \in X_{h} \subset V$ leading to a lower bound on $\left\|\operatorname{Res}\left(u_{i h}, \lambda_{i h}\right)\right\|_{-1}$.

\subsection{Meshes and discrete spaces}

We first introduce some more notation. Let henceforth $\left\{\mathcal{T}_{h}\right\}_{h}$ be a family of matching simplicial partitions of the domain $\Omega$, shape regular in the sense that the ratio of each element diameter to the diameter of its largest inscribed ball is uniformly bounded by a constant $\kappa_{\mathcal{T}}>0$. We denote by $K$ a generic element of $\mathcal{T}_{h}$. The set of vertices is denoted by $\mathcal{V}_{h}$, with interior vertices $\mathcal{V}_{h}^{\text {int }}$, vertices located on the boundary $\mathcal{V}_{h}^{\text {ext }}$, and a generic vertex a. We call $\mathcal{T}_{\mathbf{a}}$ the patch of elements of $\mathcal{T}_{h}$ which share the vertex $\mathbf{a} \in \mathcal{V}_{h}, \omega_{\mathbf{a}}$ the corresponding subdomain, and $\mathbf{n}_{\omega_{\mathbf{a}}}$ its outward unit normal. We often tacitly extend functions defined on $\omega_{\mathbf{a}}$ by zero outside of $\omega_{\mathbf{a}}$, whereas $V_{h}\left(\omega_{\mathbf{a}}\right)$ stands for the restriction of the space $V_{h}$ to $\omega_{\mathbf{a}}$. Next, $\psi_{\mathbf{a}}$ for $\mathbf{a} \in \mathcal{V}_{h}$ stands for the piecewise affine "hat" function taking value 1 at the vertex a and zero at the other vertices. Remarkably, $\left(\psi_{\mathbf{a}}\right)_{\mathbf{a} \in \mathcal{V}_{h}}$ form a partition of unity via $\sum_{\mathbf{a} \in \mathcal{V}_{h}} \psi_{\mathbf{a}}=\left.1\right|_{\Omega}$.

Let $\mathbb{P}_{s}(K), s \geq 0$, stand for polynomials of total degree at most $s$ on $K \in \mathcal{T}_{h}$, and $\mathbb{P}_{s}\left(\mathcal{T}_{h}\right)$ for piecewise polynomials on $\mathcal{T}_{h}$, without any continuity requirement. Let also $\mathbf{V}_{h} \times Q_{h} \subset \mathbf{H}(\operatorname{div}, \Omega) \times L^{2}(\Omega)$ stand for the Raviart-Thomas-Nédélec (RTN) mixed finite element spaces of for degree $p+1$, i.e., $\mathbf{V}_{h}:=\left\{\mathbf{v}_{h} \in\right.$ $\left.\mathbf{H}(\operatorname{div}, \Omega) ;\left.\mathbf{v}_{h}\right|_{K} \in\left[\mathbb{P}_{p+1}(K)\right]^{d}+\mathbb{P}_{p+1}(K) \mathbf{x}\right\}$ and $Q_{h}:=\mathbb{P}_{p+1}\left(\mathcal{T}_{h}\right)$, see Brezzi and Fortin [10] or Roberts and Thomas [52]. We also denote by $\Pi_{Q_{h}}$ the $L^{2}(\Omega)$-orthogonal projection onto $Q_{h}$.

\subsection{Equilibrated flux reconstruction for inexact solvers}

Let $\mathfrak{r}_{i h} \in \mathbb{P}_{p}\left(\mathcal{T}_{h}\right)$ be a discontinuous piecewise $p$-degree polynomial that lifts the misfit in the Galerkin orthogonality of the residual $\operatorname{Res}\left(u_{i h}, \lambda_{i h}\right)$, i.e.

$$
\left\langle\operatorname{Res}\left(u_{i h}, \lambda_{i h}\right), v_{h}\right\rangle_{V^{\prime}, V}=\lambda_{i h}\left(u_{i h}, v_{h}\right)-\left(\nabla u_{i h}, \nabla v_{h}\right)=\left(\mathfrak{r}_{i h}, v_{h}\right) \quad \forall v_{h} \in \mathbb{P}_{p}\left(\mathcal{T}_{h}\right) \cap V .
$$

A simple elementwise construction of $\mathfrak{r}_{i h}$ is proposed in [47, equation (5.2)]. Typically, $\mathfrak{r}_{i h}=0$ for an "exact" discrete algebraic solve that we do not suppose here. 
We construct $\boldsymbol{\sigma}_{i h}$ in two steps. First, solve the following homogeneous local Neumann (NeumannDirichlet close to the boundary) discrete problems on patches $\omega_{\mathbf{a}}$ :

Definition 4.1 (Equilibrated flux reconstruction). [-] For $\mathbf{a} \in \mathcal{V}_{h}$, set

$$
\begin{array}{rlrl}
\mathbf{V}_{h}^{\mathbf{a}} & :=\left\{\mathbf{v}_{h} \in \mathbf{V}_{h}\left(\omega_{\mathbf{a}}\right) ; \mathbf{v}_{h} \cdot \mathbf{n}_{\omega_{\mathbf{a}}}=0 \text { on } \partial \omega_{\mathbf{a}}\right\}, & & \mathbf{a} \in \mathcal{V}_{h}^{\text {int }}, \\
Q_{h}^{\mathbf{a}} & :=\left\{q_{h} \in Q_{h}\left(\omega_{\mathbf{a}}\right) ;\left(q_{h}, 1\right)_{\omega_{\mathbf{a}}}=0\right\}, & \\
\mathbf{V}_{h}^{\mathbf{a}} & :=\left\{\mathbf{v}_{h} \in \mathbf{V}_{h}\left(\omega_{\mathbf{a}}\right) ; \mathbf{v}_{h} \cdot \mathbf{n}_{\omega_{\mathbf{a}}}=0 \text { on } \partial \omega_{\mathbf{a}} \backslash \partial \Omega\right\}, & & \\
Q_{h}^{\mathbf{a}} & :=Q_{h}\left(\omega_{\mathbf{a}}\right), & &
\end{array}
$$

Then define $\boldsymbol{\sigma}_{i h, \text { dis }}:=\sum_{\mathbf{a} \in \mathcal{V}_{h}} \boldsymbol{\sigma}_{i h, \text { dis }}^{\mathbf{a}} \in \mathbf{V}_{h}$, where $\boldsymbol{\sigma}_{i h, \text { dis }}^{\mathbf{a}} \in \mathbf{V}_{h}^{\mathbf{a}}$ solve

$$
\boldsymbol{\sigma}_{i h, \text { dis }}^{\mathbf{a}}:=\arg \min _{\substack{\mathbf{v}_{h} \in \mathbf{V}_{h}^{\mathbf{a}}, \nabla \cdot \mathbf{v}_{h}=\Pi_{Q_{h}}\left(\lambda_{i h} u_{i h} \psi_{\mathbf{a}}-\nabla u_{i h} \cdot \nabla \psi_{\mathbf{a}}-\mathfrak{r}_{i h} \psi_{\mathbf{a}}\right)}}\left\|\psi_{\mathbf{a}} \nabla u_{i h}+\mathbf{v}_{h}\right\|_{\omega_{\mathbf{a}}} \quad \forall \mathbf{a} \in \mathcal{V}_{h}
$$

Note that the Euler-Lagrange equations for (4.3) give the standard mixed finite element formulation, cf. [22, Remark 3.7]: find $\boldsymbol{\sigma}_{i h, \text { dis }}^{\mathbf{a}} \in \mathbf{V}_{h}^{\mathbf{a}}$ and $p_{h}^{\mathbf{a}} \in Q_{h}^{\mathbf{a}}$ such that

$$
\begin{array}{rlrl}
\left(\boldsymbol{\sigma}_{i h, \mathrm{dis}}^{\mathbf{a}}, \mathbf{v}_{h}\right)_{\omega_{\mathbf{a}}}-\left(p_{h}^{\mathbf{a}}, \nabla \cdot \mathbf{v}_{h}\right)_{\omega_{\mathbf{a}}} & =-\left(\psi_{\mathbf{a}} \nabla u_{i h}, \mathbf{v}_{h}\right)_{\omega_{\mathbf{a}}} & & \forall \mathbf{v}_{h} \in \mathbf{V}_{h}^{\mathbf{a}}, \\
\left(\nabla \cdot \boldsymbol{\sigma}_{i h, \mathrm{dis}}^{\mathbf{a}}, q_{h}\right)_{\omega_{\mathbf{a}}}=\left(\lambda_{i h} u_{i h} \psi_{\mathbf{a}}-\nabla u_{i h} \cdot \nabla \psi_{\mathbf{a}}-\mathfrak{r}_{i h} \psi_{\mathbf{a}}, q_{h}\right)_{\omega_{\mathbf{a}}} & & \forall q_{h} \in Q_{h}^{\mathbf{a}} .
\end{array}
$$

Consequently, $\nabla \cdot \boldsymbol{\sigma}_{i h, \text { dis }}=\lambda_{i h} u_{i h}-\mathfrak{r}_{i h}$, cf., e.g., [46, Lemma 3.6].

Now, proceeding as in [46, Section 3.2], one can construct in a multilevel way a second flux reconstruction $\boldsymbol{\sigma}_{i h, \text { alg }} \in \mathbf{V}_{h}$ such that $\nabla \cdot \boldsymbol{\sigma}_{i h, \text { alg }}=\mathfrak{r}_{i h}$. Consequently, setting $\boldsymbol{\sigma}_{i h}:=\boldsymbol{\sigma}_{i h \text {,dis }}+\boldsymbol{\sigma}_{i h, \text { alg }}$, (4.1b) follows with $\rho_{i h}=0$. Other strategies are pursued in [21, 47], where the algebraic residual is included differently into $(4.3) /(4.4 \mathrm{~b})$. These approaches yield

$$
\nabla \cdot \boldsymbol{\sigma}_{i h, \mathrm{alg}}=\mathfrak{r}_{i h}-\rho_{i h}
$$

with $\rho_{i h} \neq 0$ and are based on precomputing some algebraic solver iterations in order to ensure that $\left\|\rho_{i h}\right\|$ is sufficiently small with respect to the two other contributions in (4.9a) below, more precisely verifying (4.9b).

\subsection{Conforming local residual liftings}

To estimate $\left\|\operatorname{Res}\left(u_{i h}, \lambda_{i h}\right)\right\|_{-1}$ from below, we solve conforming primal counterparts of problems (4.4), without the term with $\mathfrak{r}_{i h}$. On each patch $\omega_{\mathbf{a}}$ around the vertex $\mathbf{a} \in \mathcal{V}_{h}$, define

$$
\begin{array}{ll}
H_{*}^{1}\left(\omega_{\mathbf{a}}\right):=\left\{v \in H^{1}\left(\omega_{\mathbf{a}}\right) ;(v, 1)_{\omega_{\mathbf{a}}}=0\right\}, & \mathbf{a} \in \mathcal{V}_{h}^{\text {int }}, \\
H_{*}^{1}\left(\omega_{\mathbf{a}}\right):=\left\{v \in H^{1}\left(\omega_{\mathbf{a}}\right) ; v=0 \text { on } \partial \omega_{\mathbf{a}} \cap \partial \Omega\right\}, & \mathbf{a} \in \mathcal{V}_{h}^{\text {ext }},
\end{array}
$$

and let $X_{h}^{\mathbf{a}}$ be an arbitrary discrete subspace of $H_{*}^{1}\left(\omega_{\mathbf{a}}\right)$, typically $\mathbb{P}_{p+1}\left(\mathcal{T}_{\mathbf{a}}\right) \cap H_{*}^{1}\left(\omega_{\mathbf{a}}\right)$.

Definition 4.2 (Conforming local Neumann problems). Define $r_{i h}^{\mathbf{a}} \in X_{h}^{\mathbf{a}}$ by

$$
\left(\nabla r_{i h}^{\mathbf{a}}, \nabla v_{h}\right)_{\omega_{\mathbf{a}}}=\left\langle\operatorname{Res}\left(u_{i h}, \lambda_{i h}\right), \psi_{\mathbf{a}} v_{h}\right\rangle_{V^{\prime}, V} \quad \forall v_{h} \in X_{h}^{\mathbf{a}}
$$

for each $\mathbf{a} \in \mathcal{V}_{h}$. Then set $r_{i h}:=\sum_{\mathbf{a} \in \mathcal{V}_{h}} \psi_{\mathbf{a}} r_{i h}^{\mathbf{a}}$.

The functions $r_{i h}^{\mathbf{a}}$ are discrete Riesz projections of the local residual with hat-weighted test functions. As all $\psi_{\mathbf{a}} r_{i h}^{\mathbf{a}} \in H_{0}^{1}\left(\omega_{\mathbf{a}}\right), r_{i h} \in V$, though $r_{i h}^{\mathbf{a}} \notin V$.

\subsection{Dual norm of the residual equivalences}

Following Carstensen and Funken [13, Theorem 3.1], Braess et al. [8, Section 3], or [22, Lemma 3.12], there exists a constant $C_{\text {cont,PF }}$ only depending on the mesh regularity parameter $\kappa_{\mathcal{T}}$ such that

$$
\left\|\nabla\left(\psi_{\mathbf{a}} v\right)\right\|_{\omega_{\mathbf{a}}} \leq C_{\mathrm{cont}, \mathrm{PF}}\|\nabla v\|_{\omega_{\mathbf{a}}} \quad \forall v \in H_{*}^{1}\left(\omega_{\mathbf{a}}\right), \forall \mathbf{a} \in \mathcal{V}_{h}
$$


Moreover, the key result of Braess et al. [8, Theorem 7], see [23, Theorem 2.2] for three space dimensions, states that the reconstructions of Definition 4.1 satisfy the following stability property,

$$
\left\|\psi_{\mathbf{a}} \nabla u_{i h}+\boldsymbol{\sigma}_{i h, \operatorname{dis}}^{\mathbf{a}}\right\|_{\omega_{\mathbf{a}}} \leq C_{\text {st }} \sup _{v \in H_{*}^{1}\left(\omega_{\mathbf{a}}\right) ;\|\nabla v\|_{\omega_{\mathbf{a}}}=1}\left\{\left\langle\operatorname{Res}\left(u_{i h}, \lambda_{i h}\right), \psi_{\mathbf{a}} v\right\rangle_{V^{\prime}, V}-\left(\mathfrak{r}_{i h}, \psi_{\mathbf{a}} v\right)_{\omega_{\mathbf{a}}}\right\}
$$

The constant $C_{\text {st }}>0$ again only depends on $\kappa_{\mathcal{T}}$, and a computable upper bound on $C_{\text {st }}$ is given in [22, Lemma 3.23]. We can summarize the main result of this section:

Theorem 4.3 (Residual equivalences). Let $\left(u_{i h}, \lambda_{i h}\right) \in \mathbb{P}_{p}\left(\mathcal{T}_{h}\right) \cap V \times \mathbb{R}$ be arbitrary. Then, for $\boldsymbol{\sigma}_{i h, \text { dis }}$ of Definition 4.1 and $r_{i h}$ of Definition 4.2,

$$
\begin{aligned}
\left\|\operatorname{Res}\left(u_{i h}, \lambda_{i h}\right)\right\|_{-1} & \leq\left\|\nabla u_{i h}+\boldsymbol{\sigma}_{i h, \mathrm{dis}}\right\|+\left\|\boldsymbol{\sigma}_{i h, \mathrm{alg}}\right\|+\lambda_{1}^{-\frac{1}{2}}\left\|\rho_{i h}\right\|, \\
\left\|\nabla u_{i h}+\boldsymbol{\sigma}_{i h, \text { dis }}\right\|+\left\|\boldsymbol{\sigma}_{i h, \mathrm{alg}}\right\|+\lambda_{1}^{-\frac{1}{2}}\left\|\rho_{i h}\right\| & \leq 3(d+1) C_{\mathrm{st}} C_{\text {cont }, \mathrm{PF}}\left\|\operatorname{Res}\left(u_{i h}, \lambda_{i h}\right)\right\|_{-1} \\
\text { when }\left\|\boldsymbol{\sigma}_{i h, \mathrm{alg}}\right\|+\lambda_{1}^{-\frac{1}{2}}\left\|\rho_{i h}\right\| & \leq\left(2(d+1) C_{\mathrm{st}} C_{\text {cont }, \mathrm{PF}}\right)^{-1}\left\|\nabla u_{i h}+\boldsymbol{\sigma}_{i h, \mathrm{dis}}\right\|, \\
\frac{\left\langle\operatorname{Res}\left(u_{i h}, \lambda_{i h}\right), r_{i h}\right\rangle_{V^{\prime}, V}}{\left\|\nabla r_{i h}\right\|} & \leq\left\|\operatorname{Res}\left(u_{i h}, \lambda_{i h}\right)\right\|_{-1} .
\end{aligned}
$$

Proof. Fix $v \in V$ with $\|\nabla v\|=1$. Using definition (2.4a), adding and subtracting $\left(\boldsymbol{\sigma}_{i h}, \nabla v\right)$, and employing the Green theorem and the equilibrium (4.1b) yields

$$
\left\langle\operatorname{Res}\left(u_{i h}, \lambda_{i h}\right), v\right\rangle_{V^{\prime}, V}=\lambda_{i h}\left(u_{i h}, v\right)-\left(\nabla u_{i h}, \nabla v\right)=\left(\rho_{i h}, v\right)-\left(\nabla u_{i h}+\boldsymbol{\sigma}_{i h}, \nabla v\right) .
$$

Thus, definition (2.4b) of the dual norm of the residual and the Cauchy-Schwarz, Poincaré-Friedrichs, and triangle inequalities yield the bound (4.9a). This actually also holds for $\mathbf{V}_{h}$ being the cheaper RTN space of order $p$ and not $p+1$, as (4.1b) still holds. To prove (4.9b), we proceed as in [46, Theorem 7.2], while treating the weak norm $\left\|\operatorname{Res}\left(u_{i h}, \lambda_{i h}\right)\right\|_{-1}$ as in Ciarlet and Vohralík [18, Theorems 4.7 and 5.1]. One builds here crucially on inequalities (4.7) and (4.8) and relies on the choice $p+1$ for $\mathbf{V}_{h}$. Finally, the bound (4.9c) is trivial from $(2.4 \mathrm{~b})$ by taking $v=r_{i h} \in V$. Importantly, this can further be bounded from below by a Hilbertian sum of $\left\|\nabla r_{i h}^{\mathbf{a}}\right\|_{\omega_{\mathbf{a}}}$, which can be seen as in [47, proof of Theorem 2]. Thus, this bound is meaningful.

\section{Guaranteed and fully computable upper and lower bounds}

We combine here the different results of the previous sections to derive the actual guaranteed and fully computable bounds for eigenvalues (in Section 5.1) and eigenvectors (in Section 5.2). A discussion of the results is provided in Section 5.3. We will sometimes use $\zeta_{(i h)} \in V$, the solution of the Laplace source problem $-\Delta \zeta_{(i h)}=\boldsymbol{r}_{(i h)}$ in $\Omega, \zeta_{(i h)}=0$ on $\partial \Omega$, i.e.,

$$
\left(\nabla \zeta_{(i h)}, \nabla v\right)=\left(\varkappa_{i h)}, v\right) \quad \forall v \in V .
$$

We also denote by $V_{h}:=\mathbb{P}_{1}\left(\mathcal{T}_{h}\right) \cap V$ the lowest-order conforming finite element space, i.e., the span of $\psi_{\mathbf{a}}$ over all $\mathbf{a} \in \mathcal{V}_{h}^{\text {int }}$, and by $h$ the maximal diameter of all $K \in \mathcal{T}_{h}$.

\subsection{Eigenvalues}

We first tackle the upper and lower bounds for the $i$-th eigenvalue $\lambda_{i}$. We discuss the necessary auxiliary bounds below in Remark 5.3.

Theorem 5.1 (Guaranteed lower bounds for the $i$-th eigenvalue). Let the $i$-th eigenvalue, $i \geq 1$, be simple and suppose the auxiliary bounds $\underline{\lambda}_{1} \leq \lambda_{1}, \lambda_{i} \leq \bar{\lambda}_{i}, \underline{\lambda}_{i+1} \leq \lambda_{i+1}$, as well as $\lambda_{i-1} \leq \bar{\lambda}_{i-1}$ when $i>1$, for $\underline{\lambda}_{1}, \bar{\lambda}_{i}, \underline{\lambda}_{i+1}, \bar{\lambda}_{i-1}>0$. Let $\left(u_{i h}, \lambda_{i h}\right)$ be any element of $\mathbb{P}_{p}\left(\mathcal{T}_{h}\right) \cap V \times \mathbb{R}^{+}$verifying $[-]\left\|u_{i h}\right\|=1$ and the inequalities

$$
\bar{\lambda}_{i-1}<\lambda_{i h} \text { when } i>1, \quad \lambda_{i h}<\underline{\lambda}_{i+1} .
$$


Let next $\boldsymbol{\sigma}_{i h, \text { dis }}$ and $r_{i h}$ be respectively constructed following Definitions 4.1 and 4.2, let $\boldsymbol{\sigma}_{i h, \text { alg }} \in \mathbf{V}_{h}$ verify (4.5) for an inexact solver, and define

$$
\eta_{i, \mathrm{res}}:=\left\|\nabla u_{i h}+\boldsymbol{\sigma}_{i h, \mathrm{dis}}\right\|+\left\|\boldsymbol{\sigma}_{i h, \mathrm{alg}}\right\|+\underline{\lambda}_{1}^{-\frac{1}{2}}\left\|\rho_{i h}\right\| .
$$

Set

$$
\begin{aligned}
& c_{i h}:=\max \left\{\left(\frac{\lambda_{i h}}{\bar{\lambda}_{i-1}}-1\right)^{-1},\left(1-\frac{\lambda_{i h}}{\underline{\lambda}_{i+1}}\right)^{-1}\right\}, \\
& \tilde{c}_{i h}:=\max \left\{\bar{\lambda}_{i-1}^{-\frac{1}{2}}\left(\frac{\lambda_{i h}}{\bar{\lambda}_{i-1}}-1\right)^{-1}, \underline{\lambda}_{i+1}^{-\frac{1}{2}}\left(1-\frac{\lambda_{i h}}{\underline{\lambda}_{i+1}}\right)^{-1}\right\},
\end{aligned}
$$

with the left terms in the $\max$ disregarded for $i=1$. Then

$$
\left\|\nabla u_{i h}\right\|^{2}-\eta_{i}^{2} \leq \lambda_{i}
$$

where we distinguish the following three cases:

Case A (No smallness assumption) If $\left(u_{i}, u_{i h}\right) \geq 0$ is known to hold, define $\bar{\alpha}_{i h}:=\sqrt{2} \tilde{c}_{i h} \eta_{i \text {,res }}$; if only $\left(u_{i h}, \chi_{i}\right)>0$ holds, set $\bar{\alpha}_{i h}:=\sqrt{2}\left(1-\left\|u_{i h}-\Pi_{i} u_{i h}\right\|\right)^{-\frac{1}{2}} \tilde{c}_{i h} \eta_{i, \text { res }}$, where $\Pi_{i} u_{i h}$ stands for the $L^{2}(\Omega)$-orthogonal projection of $u_{i h}$ on the span of $\chi_{i}$. Then (5.4) holds with

$$
\eta_{i}^{2}:=\eta_{i, \mathrm{res}}^{2}+\left(\lambda_{i h}+\bar{\lambda}_{i}\right) \bar{\alpha}_{i h}^{2}
$$

Case B (Improved estimates under a smallness assumption) Let $\left(u_{i h}, \chi_{i}\right)>0$, define $\bar{\alpha}_{i h}:=\sqrt{2} \tilde{c}_{i h} \eta_{i, \text { res }}$ and request

$$
\bar{\alpha}_{i h} \leq \min \left\{\left(\frac{2 \underline{\lambda}_{1}}{\bar{\lambda}_{i}}\right)^{\frac{1}{2}},\left\|\chi_{i}\right\|^{-1}\left(u_{i h}, \chi_{i}\right)\right\} .
$$

Then, (5.4) holds with

$$
\eta_{i}^{2}:=c_{i h}^{2}\left(1-\frac{\bar{\lambda}_{i}}{\underline{\lambda}_{1}} \frac{\bar{\alpha}_{i h}^{2}}{4}\right)^{-1} \eta_{i, \mathrm{res}}^{2} .
$$

Case $\mathbf{C}$ (Optimal estimates under elliptic regularity assumption) Let $\left(u_{i h}, \chi_{i}\right)>0$ and assume that the solution $\zeta_{(i h)}$ of problem (5.1) belongs to the space $H^{1+\delta}(\Omega), 0<\delta \leq 1$, so that the approximation and stability estimates

$$
\begin{gathered}
\min _{v_{h} \in V_{h}}\left\|\nabla\left(\zeta_{(i h)}-v_{h}\right)\right\| \leq C_{\mathrm{I}} h^{\delta}\left|\zeta_{(i h)}\right|_{H^{1+\delta}(\Omega)}, \\
\left|\zeta_{(i h)}\right|_{H^{1+\delta}(\Omega)} \leq C_{\mathrm{S}}\left\|\boldsymbol{\varkappa}_{(i h)}\right\|
\end{gathered}
$$

are satisfied. Define $\bar{\alpha}_{i h}:=\sqrt{2} c_{i h}\left[C_{\mathrm{I}} C_{\mathrm{S}} h^{\delta} \eta_{i, \mathrm{res}}+\underline{\lambda}_{1}^{-\frac{1}{2}}\left(\left\|\boldsymbol{\sigma}_{i h, \mathrm{alg}}\right\|+\underline{\lambda}_{1}^{-\frac{1}{2}}\left\|\rho_{i h}\right\|\right)\right]$ and let

$$
\bar{\alpha}_{i h} \leq\left\|\chi_{i}\right\|^{-1}\left(u_{i h}, \chi_{i}\right)
$$

Then (5.4) holds with $\eta_{i}^{2}$ given by (5.5).

Theorem 5.2 (Improved guaranteed upper bounds for the $i$-th eigenvalue). Let the assumptions of Theorem 5.1 be satisfied, with the auxiliary bounds $\underline{\lambda}_{1} \leq \lambda_{1}, \underline{\lambda}_{i} \leq \lambda_{i} \leq \bar{\lambda}_{i}$, for $\underline{\lambda}_{1}, \underline{\lambda}_{i}, \bar{\lambda}_{i}>0$. Let also $\lambda_{i} \leq\left\|\nabla u_{i h}\right\|^{2}$, see Remark 5.4. Set

$$
\begin{aligned}
& \bar{c}_{i h}:=1 \text { if } i=1, \quad \bar{c}_{i h}:=\max \left\{\left(\frac{\lambda_{i h}}{\underline{\lambda}_{1}}-1\right)^{2}, 1\right\} \text { if } i>1, \\
& d_{i h}:=\underline{\lambda}_{i}^{2} \bar{c}_{i h}^{2}+4 \underline{\lambda}_{i} \frac{\left\langle\operatorname{Res}\left(u_{i h}, \lambda_{i h}\right), r_{i h}\right\rangle_{V^{\prime}, V}^{2}}{\left\|\nabla r_{i h}\right\|^{2}}+4 \underline{\lambda}_{i} \bar{c}_{i h}\left|\lambda_{i h}-\left\|\nabla u_{i h}\right\|^{2}\right| .
\end{aligned}
$$


Then

$$
\lambda_{i} \leq\left\|\nabla u_{i h}\right\|^{2}-\tilde{\eta}_{i}^{2}
$$

with, in Cases $A$ and $C$,

$$
\tilde{\eta}_{i}^{2}:=\max \left\{-\bar{\lambda}_{i} \bar{\alpha}_{i h}^{2}+\frac{1}{2}\left(\sqrt{d_{i h}}-\left(\underline{\lambda}_{i} \bar{c}_{i h}+2\left|\lambda_{i h}-\left\|\nabla u_{i h}\right\|^{2}\right|\right)\right), 0\right\},
$$

and, in Case $B$, for $i=1$ only,

$$
\tilde{\eta}_{1}^{2}:=\max \left\{\frac{1}{4}\left(1-\frac{\left\|\nabla u_{1 h}\right\|^{2}}{\underline{\lambda}_{2}}\right)\left(1-\frac{\bar{\alpha}_{1 h}^{2}}{4}\right)\left(\sqrt{d_{1 h}}-\left(\underline{\lambda}_{1}+2\left|\lambda_{1 h}-\left\|\nabla u_{1 h}\right\|^{2}\right|\right)\right), 0\right\} .
$$

Remark 5.3 (Auxiliary bounds $\underline{\lambda}_{1}, \underline{\lambda}_{i}$, and $\underline{\lambda}_{i+1}$ ). A straightforward consequence of the min-max principle for self-adjoint operators, see, e.g., Gilbarg and Trudinger [26], is that $\Omega \subseteq \Omega^{+} \Rightarrow \lambda_{k}\left(\Omega^{+}\right) \leq \lambda_{k}$ and $\Omega^{-} \subseteq \Omega$ $\Rightarrow \lambda_{k} \leq \lambda_{k}\left(\Omega^{-}\right)$for all $k \geq 1$, where $\lambda_{k}\left(\Omega^{ \pm}\right)$is the $k$-th eigenvalue on $\Omega^{ \pm}$. We can then obtain all $\underline{\lambda}_{1}$, $\underline{\lambda}_{i}$, and $\underline{\lambda}_{i+1}$ necessary in Theorem 5.1 by this domain inclusion for $\Omega^{+}$with known exact eigenvalues (typically rectangular d-parallelepipeds or $d$-spheres, cf. [59]). In what concerns $\underline{\lambda}_{i}$, a very precise choice is to use $\underline{\lambda}_{i}:=\left\|\nabla u_{i h}\right\|^{2}-\eta_{i}^{2}$, where $\eta_{i}^{2}$ was first computed with a rather rough bound $\underline{\lambda}_{i}$. For $\underline{\lambda}_{i+1}$, if the analytic bounds are too rough to be useful, guaranteed and easily computable numerical bounds can be used from Liu and Oishi [41] (on convex domains for $d=2$ ), Carstensen and Gedicke [16], or Liu [39], typically on a quite coarse mesh. Finally, as a "practical gratis" strategy for $\underline{\lambda}_{i+1}$, one may simply use $\lambda_{(i+1) h}$ computed by the linear algebra toolbox when solving for $\left(\lambda_{i h}, u_{i h}\right)$, see, e.g., Saad [53] and the references therein. Then Theorem 5.1 (and 5.6 below) may no longer hold stricto sensu, but sharp upper and lower bounds are still expected/observed in practice.

Remark 5.4 (Auxiliary bounds $\bar{\lambda}_{i-1}$ and $\left.\bar{\lambda}_{i}\right)$. When $\left(u_{i h}, \lambda_{i h}\right)$ is given by the conforming finite element method of Section 6 below, with an exact solver leading to satisfaction of (6.1) with $\left(u_{i h}, u_{j h}\right)=\delta_{i j}$, there holds $\lambda_{i} \leq \lambda_{i h}=\left\|\nabla u_{i h}\right\|^{2}$ and similarly $\bar{\lambda}_{i-1} \leq \lambda_{(i-1) h}=\left\|\nabla u_{(i-1) h}\right\|^{2}$, leading to rather precise auxiliary bounds $\bar{\lambda}_{i}$ and $\bar{\lambda}_{i-1}$. For the first eigenvalue, there holds $\lambda_{1} \leq\left\|\nabla u_{1 h}\right\|^{2}$ for an arbitrary $u_{1 h} \in H_{0}^{1}(\Omega)$. For the $i$-th eigenvalue, $i>1$, we in general need to resort to the $\min -\max$ principle giving

$$
\lambda_{i} \leq \max _{\boldsymbol{\xi} \in \mathbb{R}^{i},\|\boldsymbol{\xi}\|=1} \frac{\left\|\nabla \sum_{k=1}^{i} \boldsymbol{\xi}_{k} u_{k h}\right\|^{2}}{\left\|\sum_{k=1}^{i} \boldsymbol{\xi}_{k} u_{k h}\right\|^{2}}
$$

for an arbitrary linearly independent $i$-tuple $\left(u_{1 h}, \ldots, u_{i h}\right)$, where $\|\boldsymbol{\xi}\|^{2}=\sum_{k=1}^{i} \boldsymbol{\xi}_{k}^{2}$.

Remark 5.5 (Constants $C_{\mathrm{I}}$ and $C_{\mathrm{S}}$ ). Let $\Omega$ be a convex polygon in $\mathbb{R}^{2}$. Then it is classical that the solution $\zeta_{(i h)}$ of (5.1) belongs to $H^{2}(\Omega)$ and $\left|\zeta_{(i h)}\right|_{H^{2}(\Omega)}=\left\|\Delta \zeta_{(i h)}\right\|=\left\|\boldsymbol{r}_{(i h)}\right\|$, so that $\delta=1$ and $C_{\mathrm{S}}=1$, see Grisvard [28, Theorem 4.3.1.4]. In this situation, calculable bounds on $C_{\mathrm{I}}$ can be found in Liu and Kikuchi [40] and Carstensen et al. [17], see also Liu and Oishi [41, Section 2] and the references therein; in particular, for a mesh formed by isosceles right-angled triangles, $C_{\mathrm{I}} \leq \frac{0.493}{\sqrt{2}}$.

We now prove Theorems 5.1 and 5.2, separately for each case:

Proof (Case A). 1) Lower bound of Theorem 5.1. If $\left(u_{i}, u_{i h}\right) \geq 0$ is known to hold, we can start from the $L^{2}(\Omega)$ bound (3.10). If this is not the case but $\left(u_{i h}, \chi_{i}\right)>0$ holds, we first inspect the proof of Lemma 3.2 to obtain an alternative $L^{2}(\Omega)$ estimate. We have $-2\left(u_{i}, u_{i h}\right)=-2\left(u_{i}, u_{i h}-\Pi_{i} u_{i h}\right)-2\left(u_{i}, \Pi_{i} u_{i h}\right)$. Note that the second term is negative by the sign assumption $\left(u_{i}, \chi_{i}\right)>0$ on $u_{i}$. So, instead of $(3.8)$, as $\left\|u_{i}\right\|=1$ and $\left\|u_{i h}-\Pi_{i} u_{i h}\right\|<1$,

$$
\left\|u_{i}-u_{i h}\right\|^{2} \leq 2+2\left\|u_{i h}-\Pi_{i} u_{i h}\right\|=: \delta_{i h}<4 .
$$

Consequently, the quadratic inequality in the proof of Lemma 3.2 implies $\left\|u_{i}-u_{i h}\right\|^{2} \leq\left\|\nabla \boldsymbol{\varkappa}_{(i h)}\right\|^{2} \widetilde{C}_{i h}^{-1}(1-$ $\left.\delta_{i h} / 4\right)^{-1}$. Thus, the bound (4.9a) and assumption (5.2) enable us to give a computable upper bound on the $L^{2}(\Omega)$ error by the estimator $\bar{\alpha}_{i h}$; note that $\min \{a, b\}^{-\frac{1}{2}}=\max \left\{a^{-\frac{1}{2}}, b^{-\frac{1}{2}}\right\}$, linking the constant $\widetilde{C}_{i h}$ of (3.9) with $\tilde{c}_{i h}$ of (5.3b). Consequently, the bound in (5.4) follows by combining the upper bounds in (3.12), (3.18a), and once again (4.9a). 
2) Upper bound of Theorem 5.2. We start from the lower bound in (3.12). We then need to bound $\left\|\nabla\left(u_{i}-u_{i h}\right)\right\|^{2}$ from below, for which we use (3.18b). Relying on the simplifying assumption $\lambda_{i} \leq\left\|\nabla u_{i h}\right\|^{2}$, satisfied namely in cases discussed in Remark 5.4, $\gamma_{i h}$ of (3.17) simplifies to $\left\|\nabla\left(u_{i}-u_{i h}\right)\right\|^{2}$. Thus (3.18b) forms a quadratic inequality for $\left\|\nabla\left(u_{i}-u_{i h}\right)\right\|^{2}$, yielding, in combination with (4.9c),

$$
\left\|\nabla\left(u_{i}-u_{i h}\right)\right\|^{2} \geq \frac{1}{2}\left(\sqrt{d_{i h}}-\left(\underline{\lambda}_{i} \bar{c}_{i h}+2\left|\lambda_{i h}-\left\|\nabla u_{i h}\right\|^{2}\right|\right)\right) .
$$

Thus (5.11) with the estimator (5.12) follows.

Proof (Case B). The proof proceeds as above. Note that conditions in (5.6) imply that $\alpha_{i h} \leq \sqrt{2 \frac{\lambda_{1}}{\lambda_{i}}}$ and $\alpha_{i h} \leq\left\|\chi_{i}\right\|^{-1}\left(u_{i h}, \chi_{i}\right)$ for $\alpha_{i h}$ of (3.10). We can thus use Lemma 3.3 to find that $\left(u_{i}, u_{i h}\right)$ is indeed nonnegative, Lemma 3.2 for the $L^{2}(\Omega)$ bound, and the improved estimates (3.19) of Theorem 3.5 and (3.13) of Theorem 3.4. For the latter, that seems only to hold for the first eigenpair, we also employ the inequality $1-\frac{\left\|\nabla u_{1 h}\right\|^{2}}{\underline{\lambda}_{2}} \leq 1-\frac{\lambda_{1}}{\lambda_{2}}$ and (5.14) for $i=1$.

Proof (Case C). The proof is as in Case A (with $\left(u_{i}, u_{i h}\right) \geq 0$ ), but it relies on Lemma 3.1 instead of Lemma 3.2. It additionally uses the Aubin-Nitsche trick, cf. [9, Theorem 5.4.8], [28, Theorem 4.3.1.4], or [6]. By (5.1), (2.5a), and (4.2)

$$
\left\|\boldsymbol{\varkappa}_{(i h)}\right\|^{2}=\left(\nabla \zeta_{(i h)}, \nabla \boldsymbol{\varkappa}_{(i h)}\right)=\left(\nabla\left(\zeta_{(i h)}-\zeta_{i h}\right), \nabla \boldsymbol{\varkappa}_{(i h)}\right)+\left(\mathfrak{r}_{i h}, \zeta_{i h}\right),
$$

where $\zeta_{i h} \in V_{h}$ is the minimizer in (5.8a). Employing (4.5), the Green theorem, the Poincaré-Friedrichs inequality $\left\|\zeta_{i h}\right\| \leq \underline{\lambda}_{1}^{-\frac{1}{2}}\left\|\nabla \zeta_{i h}\right\|$, and the stability $\left\|\nabla \zeta_{i h}\right\| \leq\left\|\nabla \zeta_{(i h)}\right\|$

$$
\left(\mathfrak{r}_{i h}, \zeta_{i h}\right)=-\left(\boldsymbol{\sigma}_{i h, \text { alg }}, \nabla \zeta_{i h}\right)+\left(\rho_{i h}, \zeta_{i h}\right) \leq\left(\left\|\boldsymbol{\sigma}_{i h, \text { alg }}\right\|+\underline{\lambda}_{1}^{-\frac{1}{2}}\left\|\rho_{i h}\right\|\right)\left\|\nabla \zeta_{(i h)}\right\| .
$$

Noting that (5.1) gives $\left\|\nabla \zeta_{(i h)}\right\| \leq \underline{\lambda}_{1}^{-\frac{1}{2}}\left\|\boldsymbol{\iota}_{i h)}\right\|$, the Cauchy-Schwarz inequality, estimates (5.8), and the characterization $(2.5 \mathrm{~b})$ altogether give

$$
\left\|\boldsymbol{\iota}_{i h)}\right\| \leq C_{\mathrm{I}} C_{\mathrm{S}} h^{\delta}\left\|\operatorname{Res}\left(u_{i h}, \lambda_{i h}\right)\right\|_{-1}+\underline{\lambda}_{1}^{-\frac{1}{2}}\left(\left\|\boldsymbol{\sigma}_{i h, \mathrm{alg}}\right\|+\underline{\lambda}_{1}^{-\frac{1}{2}}\left\|\rho_{i h}\right\|\right) .
$$

\subsection{Eigenvectors}

We now summarize our estimate on the energy error in the approximation of the $i$-th eigenvector, as well as its efficiency and robustness:

Theorem 5.6 (Guaranteed and robust bound for the $i$-th eigenvector error). Let the assumptions of Theorem 5.1 be verified. Then the energy error can be bounded via

$$
\left\|\nabla\left(u_{i}-u_{i h}\right)\right\| \leq \eta_{i},
$$

where $\eta_{i}$ is defined in the Cases $A$ and $C$ by (5.5) and in Case B by (5.7), with appropriate $\bar{\alpha}_{i h}$. Under condition (4.9b), all these estimators $\eta_{i}$ are efficient as

$$
\eta_{i, \text { res }}^{2} \leq 3^{2}(d+1)^{2} C_{\mathrm{st}}^{2} C_{\mathrm{cont}, \mathrm{PF}}^{2}\left(\frac{\left(\left|\lambda_{i h}-\left\|\nabla u_{i h}\right\|^{2}\right|+\gamma_{i h}\right)^{2}}{\lambda_{i}}+\bar{C}_{i h}\left\|\nabla\left(u_{i}-u_{i h}\right)\right\|^{2}\right) .
$$

Proof. The guaranteed error bound (5.15) follows as in Theorem 5.1 upon combining the upper bounds in estimates (3.18) or (3.19) together with (4.9a). The efficiency (5.16) is a consequence of (4.9b) and of $(3.18 \mathrm{~b})$. 


\subsection{Comments}

We collect here comments about Theorems 5.1, 5.2, and 5.6.

Remark 5.7 (Inexact solvers). The results of Theorems 5.1, 5.2, and 5.6 are presented in a general context of an inexact algebraic solver. Shall the algebraic residual representer $\mathfrak{r}_{i h}$ in (4.2) be zero and the Rayleigh quotient link $\left\|\nabla u_{i h}\right\|^{2}=\lambda_{i h}$ hold, many simplifications apply. Namely, $\boldsymbol{\sigma}_{i h, \text { alg }}=\mathbf{0}, \rho_{i h}=0$, and $\left\|\nabla u_{i h}\right\|^{2}$ can be systematically replaced by $\lambda_{i h}$, making in particular disappear numerous terms in the estimators $\eta_{i}$ and $\tilde{\eta}_{i}$. The polynomial-degree-robust efficiency (5.16) holds under the condition (4.9b) only, which is a typical inexactness (stopping) criterion. For the elliptic regularity Case $C$, though, it appears wise to only stop the iterations when $\underline{\lambda}_{1}^{-\frac{1}{2}}\left(\left\|\boldsymbol{\sigma}_{i h, \text { alg }}\right\|+\underline{\lambda}_{1}^{-\frac{1}{2}}\left\|\rho_{i h}\right\|\right)$ is comparable to the first term in $\bar{\alpha}_{i h}$.

Remark 5.8 (Sharpness and comparison of the different bounds of Theorems 5.1 and 5.6). The advantage of Case $A$ is that it holds on an arbitrarily coarse mesh, provided that only the structural assumption (5.2) holds. It may, however, lead to a larger overestimation of the error. Case B, under the "fine enough mesh" condition (5.6), then significantly improves the multiplicative factor in front of the central term $\eta_{i, \text { res }}=\left\|\nabla u_{i h}+\boldsymbol{\sigma}_{i h, \text { dis }}\right\|+\left\|\boldsymbol{\sigma}_{i h, \text { alg }}\right\|+\underline{\lambda}_{1}^{-\frac{1}{2}}\left\|\rho_{i h}\right\|$, in limit to the factor $c_{i h}$ given by (5.3a). The bound of Case $B$ still holds without any regularity/convexity/dimension assumption and all the quantities appearing are known. Finally, also the factor $c_{i h}$ is asymptotically removed in Case $C$, when $\delta>0$ and $h \rightarrow 0$. Here, however, elliptic regularity is needed, see Remark 5.5.

Remark 5.9 (Dependence on the maximal element diameter $h$ ). The maximal element diameter $h$ is not present at all in Cases $A$ and $B$ of Theorem 5.1 and it does not necessarily need to tend to zero in Case C: it only appears as a multiplicative factor of the principal estimator $\eta_{i, \mathrm{res}}$. [-] This stands in contrast to previous guaranteed results like [41, Theorem 4.3], [16, Theorem 3.2], or [39, Theorem 2.1].

Remark 5.10 (Polynomial-degree robustness). The multiplicative factor in the parenthesis in (5.16) takes the form $\left\|\nabla\left(u_{i}-u_{i h}\right)\right\|^{2}\left(\bar{C}_{i h}+\frac{\left\|\nabla\left(u_{i}-u_{i h}\right)\right\|^{2}}{\lambda_{i}}\right)$ for an exact algebraic solver in the context of the finite element method (6.1) below. Noting that $\frac{\left\|\nabla\left(u_{i}-u_{i h}\right)\right\|^{2}}{\lambda_{i}} \leq \frac{2\left(\lambda_{i}+\lambda_{i h}\right)}{\lambda_{i}}$ (in fact this term becomes negligible with mesh refinement/increasing the polynomial degree), we conclude that the result of Theorem 5.6 is fully robust with respect to the polynomial degree $p$ of $u_{i h}$ : all the constants in the comparison between the error $\left\|\nabla\left(u_{i}-u_{i h}\right)\right\|$ and the estimate featuring $\left\|\nabla u_{i h}+\sigma_{i h, \text { dis }}\right\|$ are independent of $p$. Note, though, that the factor $\bar{C}_{i h}$ given by (3.16) deteriorates for higher eigenvalues.

Remark 5.11 (Error localization and mesh adaptivity). Since there holds $\eta_{i, \mathrm{res}}^{2} \leq 3 \sum_{K \in \mathcal{T}_{h}}\left(\| \nabla u_{i h}+\right.$ $\left.\boldsymbol{\sigma}_{i h, \mathrm{dis}}\left\|_{K}^{2}+\right\| \boldsymbol{\sigma}_{i h, \mathrm{alg}}\left\|_{K}^{2}+\underline{\lambda}_{1}^{-1}\right\| \rho_{i h} \|_{K}^{2}\right)$, a crucial advantage of the estimators of Theorems 5.1 and 5.6 is that these local contributions can be used in adaptive mesh refinement based on marking strategies. This is tightly linked to Remark 5.9.

\section{Application to conforming finite elements}

We verify in this section the conditions of the application of our results to the conforming finite element method.

Let $V_{h}:=\mathbb{P}_{p}\left(\mathcal{T}_{h}\right) \cap V$ for a given polynomial degree $p \geq 1$. In the finite element method, the exact $i$-th eigenpair $\left(u_{i h}, \lambda_{i h}\right) \in V_{h} \times \mathbb{R}^{+}$is such that $\left(u_{i h}, u_{j h}\right)=\delta_{i j}, 1 \leq i, j \leq \operatorname{dim} V_{h}$, and

$$
\left(\nabla u_{i h}, \nabla v_{h}\right)=\lambda_{i h}\left(u_{i h}, v_{h}\right) \quad \forall v_{h} \in V_{h},
$$

with the signs ideally fixed by $\left(u_{i}, u_{i h}\right) \geq 0$, practically by $\left(u_{i h}, \chi_{i}\right)>0$. Thus, upon verifying $(5.2)$ and possibly checking (5.6) or (5.9), all the results of Theorems 5.1, 5.2, and 5.6 hold for any $p \geq 1$. Note that an inexact solution of (6.1) in the form (4.2) is taken into account. Shall (6.1) hold, $\mathfrak{r}_{i h}$ in (4.2) vanishes and, moreover, choosing $v_{h}=u_{i h}$ in (6.1) yields $\left\|\nabla u_{i h}\right\|^{2}=\lambda_{i h}$. 


\begin{tabular}{rrrrrr}
\hline & $N$ & $h$ & ndof & $\underline{\lambda}_{2}-\lambda_{1 h}(5.2)$ & $\left\|\chi_{1}\right\|^{-1}\left(u_{1 h}, \chi_{1}\right)-\bar{\alpha}_{1 h}(5.9)$ \\
\hline$\underline{\lambda}_{1}=1.5 \pi^{2}$ & 3 & 0.4714 & 16 & $19.04(\checkmark)$ & $-0.64(\times)$ \\
$\underline{\lambda}_{2}=4.5 \pi^{2}$ & 4 & 0.3536 & 25 & $21.55(\checkmark)$ & $0.12(\checkmark)$ \\
& 5 & 0.2828 & 36 & $22.69(\checkmark)$ & $0.40(\checkmark)$ \\
\hline$\underline{\lambda}_{1}=0.5 \pi^{2}$ & 3 & 0.4714 & 16 & $4.233(\checkmark)$ & $-3.49(\times)$ \\
$\underline{\lambda}_{2}=3 \pi^{2}$ & 4 & 0.3536 & 25 & $6.743(\checkmark)$ & $-0.66(\times)$ \\
& 5 & 0.2828 & 36 & $7.887(\checkmark)$ & $0.02(\checkmark)$ \\
\hline
\end{tabular}

Table 1: [Unit square, structured mesh] Validation of assumptions (5.2) and (5.9)

\section{Numerical experiments}

We finally numerically illustrate the estimates of Theorems $5.1,5.2$, and 5.6 on three test cases in $\mathbb{R}^{2}$, for conforming finite elements (6.1) of order $p=1$. We actually only use the cheaper Raviart-Thomas-Nédélec space of degree $p=1$ for the flux equilibration instead of $p+1$. This still gives guaranteed bounds, see the proof of Theorem 4.3, and we do not observe any asymptotic loss of efficiency. The implementation was done in the FreeFem ++ code [30]. When we only consider one eigenvalue, it is implicitly assumed that we have chosen $\chi_{1}=1$ for the sign characterization. We consider five test settings with an exact solver and illustrate the use of an inexact solver in a sixth one.

\subsection{First eigenvalue on the unit square}

We start by testing the framework on a unit square $\Omega=(0,1)^{2}$ and focus on the first eigenvalue. The eigenvalues on a square of size $H$ being $\pi^{2}\left(k^{2}+l^{2}\right) / H^{2}, k, l=1, \ldots, \infty$, the first and second eigenvalues are $\lambda_{1}=2 \pi^{2}$ and $\lambda_{2}=5 \pi^{2}$, respectively. In consequence, we can easily choose different $\underline{\lambda}_{1} \leq \lambda_{1}$ and $\underline{\lambda}_{2} \leq \lambda_{2}$ for the auxiliary eigenvalue bounds and analyze the sensitivity of our results with respect to these choices. The first eigenfunction is given by $u_{1}(x, y)=\sin (\pi x) \sin (\pi y)$. We focus here on the refined elliptic regularity of Case $\mathrm{C}$, since $d=2$ and the domain is convex, with constants $C_{\mathrm{S}}=1$ and $\delta=1$ given in Remark 5.5.

\subsubsection{Structured mesh}

We first illustrate in Table 1 how quickly the computable conditions (5.2) and (5.9) are satisfied under a uniform refinement of a structured mesh. We take $C_{\mathrm{I}}=\frac{0.493}{\sqrt{2}}$ following Remark 5.5 and consider $N=3,4,5$ subdivisions of each boundary of $\Omega$ for the two choices $\underline{\lambda}_{1}=1.5 \pi^{2}, \underline{\lambda}_{2}=4.5 \pi^{2}$ and $\underline{\lambda}_{1}=0.5 \pi^{2}, \underline{\lambda}_{2}=3 \pi^{2}$, respectively. Note that the finite element space on the coarsest mesh such that all conditions are satisfied contains 25, respectively 36, degrees of freedom only. Indeed, it turns out that our conditions are rather mild.

Next, Figure 1 (left) illustrates the convergence of the error $\lambda_{1 h}-\lambda_{1}$ as well as of its lower and upper bounds $\tilde{\eta}_{1}^{2}, \eta_{1}^{2}$ given by Case $\mathrm{C}$ of Theorems 5.1 and 5.2. We also plot the energy error in the eigenfunction $\left\|\nabla\left(u_{1}-u_{1 h}\right)\right\|$ and its upper bound $\eta_{1}$ of Theorem 5.6, Case C. The convergence rates are optimal as expected from the theory.

We present in Table 2 precise numbers of the lower and upper bounds $\lambda_{1 h}-\eta_{1}^{2} \leq \lambda_{1} \leq \lambda_{1 h}-\tilde{\eta}_{1}^{2}$ on the exact eigenvalue $\lambda_{1}$, the effectivity indices of the lower and upper bounds $\tilde{\eta}_{1}^{2} \leq \lambda_{1 h}-\lambda_{1} \leq \eta_{1}^{2}$ of the error $\lambda_{1 h}-\lambda_{1}$, and the effectivity index of the upper bound $\left\|\nabla\left(u_{1}-u_{1 h}\right)\right\| \leq \eta_{1}$, given respectively by

$$
I_{\lambda, \mathrm{eff}}^{\mathrm{lb}}:=\frac{\lambda_{1 h}-\lambda_{1}}{\tilde{\eta}_{1}^{2}}, \quad I_{\lambda, \mathrm{eff}}^{\mathrm{ub}}:=\frac{\eta_{1}^{2}}{\lambda_{1 h}-\lambda_{1}}, \quad I_{u, \mathrm{eff}}^{\mathrm{ub}}:=\frac{\eta_{1}}{\left\|\nabla\left(u_{1}-u_{1 h}\right)\right\|} .
$$

We observe rather sharp results, and this also for the relative size of the first eigenvalue confidence interval

$$
E_{\lambda, \mathrm{rel}}:=2 \frac{\left(\lambda_{1 h}-\tilde{\eta}_{1}^{2}\right)-\left(\lambda_{1 h}-\eta_{1}^{2}\right)}{\left(\lambda_{1 h}-\tilde{\eta}_{1}^{2}\right)+\left(\lambda_{1 h}-\eta_{1}^{2}\right)}
$$



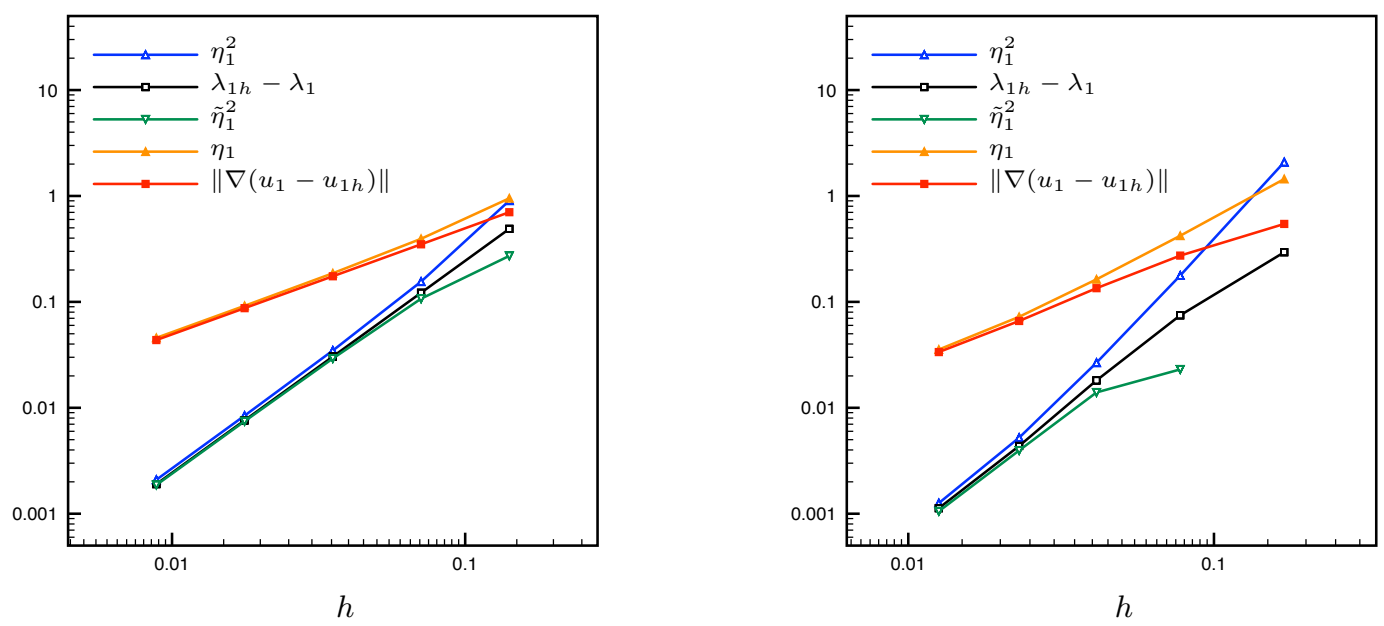

Figure 1: [Unit square] Error in the eigenvalue and eigenvector approximation, its lower bound (eigenvalue only), and its upper bound for the choice $\underline{\lambda}_{1}=1.5 \pi^{2}, \underline{\lambda}_{2}=4.5 \pi^{2}$; sequence of structured (left) and unstructured but quasi-uniform (right) meshes; Case $\mathrm{C}$

\begin{tabular}{rcrccrrrrrr}
\hline \multicolumn{1}{r|}{$N$} & \multicolumn{1}{c}{$h$} & \multicolumn{1}{c}{ ndof } & \multicolumn{1}{c}{$\lambda_{1}$} & $\lambda_{1 h}$ & $\lambda_{1 h}-\eta_{1}^{2}$ & $\lambda_{1 h}-\tilde{\eta}_{1}^{2}$ & $I_{\lambda, \text { eff }}^{\text {lb }}$ & $I_{\lambda, \text { eff }}^{\mathrm{ub}}$ & $E_{\lambda, \text { rel }}$ & $I_{u, \text { eff }}^{\mathrm{ub}}$ \\
\hline 10 & 0.1414 & 121 & 19.7392 & 20.2284 & 19.3256 & 19.9566 & 1.80 & 1.85 & $3.21 \mathrm{e}-02$ & 1.35 \\
20 & 0.0707 & 441 & 19.7392 & 19.8611 & 19.7058 & 19.7539 & 1.14 & 1.27 & $2.44 \mathrm{e}-03$ & 1.13 \\
40 & 0.0354 & 1681 & 19.7392 & 19.7697 & 19.7349 & 19.7404 & 1.04 & 1.14 & $2.79 \mathrm{e}-04$ & 1.07 \\
80 & 0.0177 & 6561 & 19.7392 & 19.7468 & 19.7384 & 19.7394 & 1.02 & 1.11 & $4.91 \mathrm{e}-05$ & 1.05 \\
160 & 0.0088 & 25921 & 19.7392 & 19.7411 & 19.7390 & 19.7392 & 1.02 & 1.10 & $1.14 \mathrm{e}-05$ & 1.05 \\
\hline
\end{tabular}

Table 2: [Unit square, structured mesh] Lower and upper bounds on the exact eigenvalue $\lambda_{1}$, the effectivity indices, and size of the relative $\lambda_{1}$ confidence interval; $\underline{\lambda}_{1}=1.5 \pi^{2}, \underline{\lambda}_{2}=4.5 \pi^{2}$; Case C

\subsubsection{Unstructured mesh}

Consider now a sequence of unstructured quasi-uniform meshes, obtained by an initial partition of each boundary edge into $N$ intervals. Conditions (5.2) and (5.9) turn here to be satisfied similarly as in Table 1.

The convergence plots for this case are presented in Figure 1 (right), showing a similar behavior as for the structured meshes. This time, we use the upper bound on $C_{\mathrm{I}}$ according to [40, Eqn. (46)]: $C_{\mathrm{I}}=$ $0.493 \max _{K \in \mathcal{T}_{h}} \frac{1+\left|\cos \left(\theta_{K}\right)\right|}{\sin \left(\theta_{K}\right)} \sqrt{\frac{\nu_{+}\left(\alpha_{K}, \theta_{K}\right)}{2}} \frac{h_{K}^{[40]}}{h_{K}}$. We refer to [40] for the definition of $h_{K}^{[40]}$ and other notation. We observe in Table 3 that the results are similar to structured meshes; in particular the case of $\lambda_{1}=0.5 \pi^{2}$, $\underline{\lambda}_{2}=3 \pi^{2}$ is less sensitive to the unstructured mesh (not presented).

\subsection{First eigenvalue on an L-shaped domain: mesh adaptivity}

We next consider the L-shaped domain $\Omega:=(-1,1)^{2} \backslash[0,1] \times[-1,0]$, where $\lambda_{1} \approx 9.6397238440$ is known to high accuracy [59]. Including $\Omega$ into the square $\Omega^{+}=(-1,1)^{2}$, cf. Remark 5.3, we take $\underline{\lambda}_{1}=\lambda_{1}\left(\Omega^{+}\right)=\pi^{2} / 2$, whereas $\underline{\lambda}_{2}=15.1753$ from Table 1 of [39] is employed. We test here the Cases A and B within an adaptive refinement strategy. To do so, we use the local character of our estimators, see Remark 5.11. We employ the Dörfler marking with $\theta=0.6$ and the newest vertex bisection mesh refinement.

Table 4 illustrates whether the conditions (5.2) and (5.6) are satisfied under this adaptive refinement. Figure 2 (right) illustrates the error in the eigenvalue and the eigenvector and their bounds (5.4), (5.11), and (5.15). Optimal convergence rates are indicated by dashed lines. The initial mesh is structured with 22 degrees of freedom and the conditions (5.2) and (5.6) are all satisfied starting from 140 degrees of freedom. The transition from Case A to Case B in Theorems 5.1, 5.2, and 5.6 is marked by a dotted line. Figure 2 (left) then depicts an adaptively refined mesh and Table 5 presents more details on the errors and efficiencies. 


\begin{tabular}{rrrrrrrrrrr}
\hline \multicolumn{1}{r}{$N$} & \multicolumn{1}{c}{$h$} & \multicolumn{1}{c}{ ndof } & \multicolumn{1}{c}{$\lambda_{1}$} & $\lambda_{1 h}$ & $\lambda_{1 h}-\eta_{1}^{2}$ & $\lambda_{1 h}-\tilde{\eta}_{1}^{2}$ & $I_{\lambda, \text { eff }}^{\mathrm{lb}}$ & $I_{\lambda, \text { eff }}^{\mathrm{ub}}$ & $E_{\lambda, \text { rel }}$ & $I_{u, \text { eff }}^{\mathrm{ub}}$ \\
\hline 10 & 0.1698 & 143 & 19.7392 & 20.0336 & 17.9458 & 20.6491 & - & 7.09 & $1.40 \mathrm{e}-01$ & 2.65 \\
20 & 0.0776 & 523 & 19.7392 & 19.8139 & 19.6366 & 19.7909 & 3.24 & 2.37 & $7.83 \mathrm{e}-03$ & 1.54 \\
40 & 0.0413 & 1975 & 19.7392 & 19.7573 & 19.7307 & 19.7434 & 1.30 & 1.47 & $6.42 \mathrm{e}-04$ & 1.21 \\
80 & 0.0230 & 7704 & 19.7392 & 19.7436 & 19.7383 & 19.7396 & 1.10 & 1.20 & $6.41 \mathrm{e}-05$ & 1.09 \\
160 & 0.0126 & 30666 & 19.7392 & 19.7403 & 19.7391 & 19.7393 & 1.07 & 1.12 & $1.04 \mathrm{e}-05$ & 1.06 \\
\hline
\end{tabular}

Table 3: [Unit square, unstructured mesh] Lower and upper bounds on the exact eigenvalue $\lambda_{1}$, the effectivity indices, and size of the relative $\lambda_{1}$ confidence interval; $\underline{\lambda}_{1}=1.5 \pi^{2}, \underline{\lambda}_{2}=4.5 \pi^{2}$; Case C

\begin{tabular}{rcrrrr}
\hline Level & $h$ & ndof & $\underline{\lambda}_{2}-\lambda_{1 h}(5.2)$ & $\bar{\alpha}_{1 h} \sqrt{\lambda_{1 h} / 2 \underline{\lambda}_{1}}(5.6)$ & $\left\|\chi_{1}\right\|^{-1}\left(u_{1 h}, \chi_{1}\right)-\bar{\alpha}_{1 h}(5.6)$ \\
\hline 1 & 0.7500 & 22 & $1.8223(\times)$ & $2.97(\times)$ & $-6.17(\times)$ \\
4 & 0.7071 & 34 & $3.8799(\checkmark)$ & $0.94(\checkmark)$ & $-1.27(\times)$ \\
10 & 0.5000 & 140 & $5.2053(\checkmark)$ & $0.33(\checkmark)$ & $0.13(\checkmark)$ \\
\hline
\end{tabular}

Table 4: [L-shaped domain, adaptive mesh refinement] Validation of the assumptions (5.2) and (5.6) for $\underline{\lambda}_{1}=\pi^{2} / 2$ and $\underline{\lambda}_{2}=15.1753$

\subsection{First eigenvalue on a domain with a hole: mesh adaptivity}

We next consider a domain with a polygonal hole, see Figure 3 (left) illustrating the mesh used at iteration 20 of our adaptive mesh refinement strategy. The lower bounds $\underline{\lambda}_{1}$ and $\underline{\lambda}_{2}$ on the first and second eigenvalue have been obtained once and for all before starting the adaptive algorithm following the estimates derived in [39], on a uniform mesh with 1143 nodes. Figure 3 (right) shows the interval between our lower $\left(\lambda_{1 h}-\eta_{1}^{2}\right)$ and upper $\left(\lambda_{1 h}-\tilde{\eta}_{1}^{2}\right)$ bounds on the first eigenvalue, relying on Case B of Theorems 5.1 and 5.2, whose assumptions hold starting from 2494 degrees of freedom; Table 6 states the numbers. Note that the interval size $\left(\lambda_{1 h}-\tilde{\eta}_{1}^{2}\right)-\left(\lambda_{1 h}-\eta_{1}^{2}\right)=\eta_{1}^{2}-\tilde{\eta}_{1}^{2}$ behaves like $1 /$ ndof .

\subsection{Higher eigenvalues}

We now test the upper and lower bounds for higher eigenvalues. First we consider the unit triangle with vertices $(0,0),(1,0),(0,1)$ and a family of structured meshes. The auxiliary lower bounds are obtained by a computation on a fixed coarse mesh with 2145 triangles following [39], which results in

$$
\underline{\lambda}_{1}=49.2883, \quad \underline{\lambda}_{2}=98.4296, \quad \underline{\lambda}_{3}=127.937, \quad \underline{\lambda}_{4}=166.975, \quad \underline{\lambda}_{5}=196.439 .
$$

Figure 4 gives the convergence plots for the first four eigenvalues and Table 7 provides more details on absolute numbers and efficiency. As the domain is convex (case C), we obtain excellent upper bounds for the error in all four eigenvalue/eigenvector pairs. The lower bound of the eigenvalue error (the improved eigenvalue upper bound of Theorem 5.2) is, however, degrading for higher eigenvalues.

We now apply the same setting to the L-shaped domain where we obtain again the auxiliary lower bounds by the method presented in [39] for a coarse structured mesh with 3201 triangles resulting in

$$
\underline{\lambda}_{1}=9.60692, \quad \underline{\lambda}_{2}=15.1695, \quad \underline{\lambda}_{3}=19.6932, \quad \underline{\lambda}_{4}=29.4166, \quad \underline{\lambda}_{5}=31.7363 \text {. }
$$

Figure 5 plots the convergence of the errors the estimators whereas Table 8 provides more details on the efficiency. We now observe that the efficiency also degrades for the upper bound of the eigenvalue and eigenvector error. Further, improved lower bounds of the eigenvalue error are not available for the considered meshes for $i>1$. This appears as the resulting $\tilde{\eta}_{i}$ are all equal to zero, see (5.12), respectively (5.13), so that our eigenvalue upper bound stays that of the finite element method. For all meshes and all considered eigenvalues, though, our estimates still give a rather tight guaranteed eigenvalue confidence interval and quite reasonable eigenvector effectivity indices. We can also observe by a jump of the blue curve $\left(\eta_{i}^{2}\right)$ the change between the cases $\mathrm{A}$ and $\mathrm{B}$. The critical mesh size where this change occurs seems to degrade with increasing eigenvalues. 

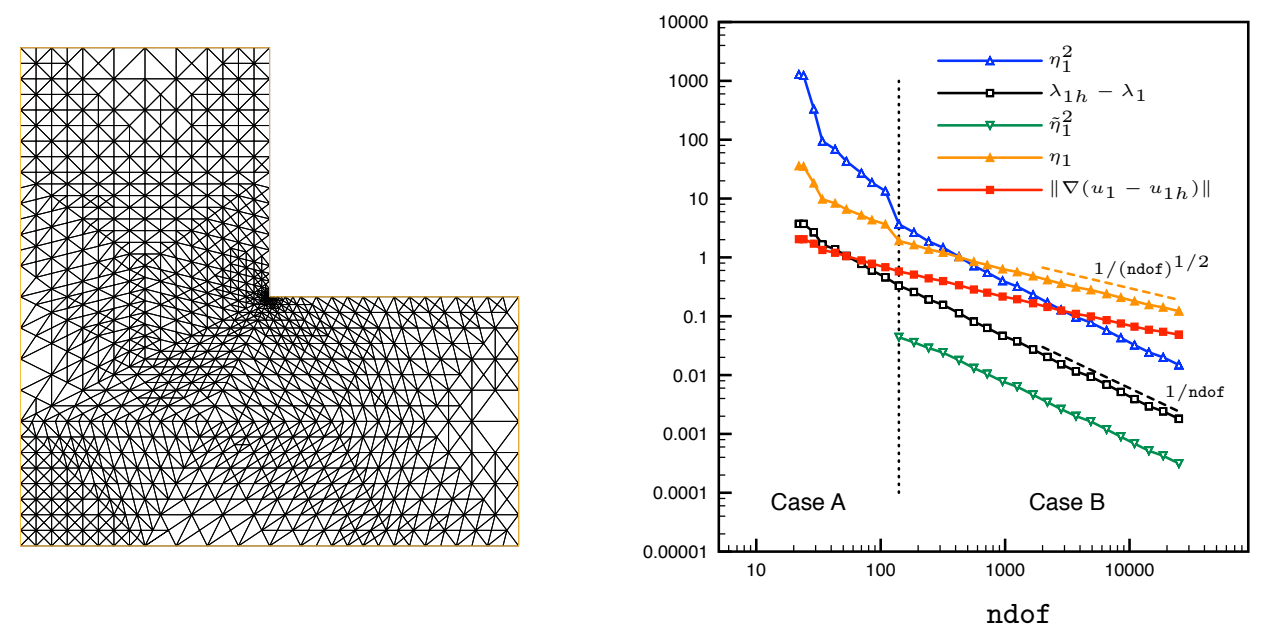

Figure 2: [L-shaped domain, adaptive mesh refinement] Mesh of the adaptive algorithm on step 18 (left) and error in the first eigenvalue and eigenvector approximation, its lower bound (eigenvalue only), and its upper bound (right); Cases A and B

\begin{tabular}{rrccrrrrrr}
\hline Level & \multicolumn{1}{c}{ ndof } & $\lambda_{1}$ & $\lambda_{1 h}$ & $\lambda_{1 h}-\eta_{1}^{2}$ & $\lambda_{1 h}-\tilde{\eta}_{1}^{2}$ & $I_{\lambda, \text { eff }}^{\text {lb }}$ & $I_{\lambda, \text { eff }}^{\text {ub }}$ & $E_{\lambda, \text { rel }}$ & $I_{u, \text { eff }}^{\text {ub }}$ \\
\hline 10 & 140 & 9.6397 & 9.9700 & 6.3175 & 9.9260 & 7.50 & 11.06 & $4.44 \mathrm{e}-01$ & 3.31 \\
15 & 561 & 9.6397 & 9.7207 & 9.0035 & 9.7075 & 6.17 & 8.86 & $7.53 \mathrm{e}-02$ & 2.98 \\
20 & 2188 & 9.6397 & 9.6601 & 9.4887 & 9.6566 & 5.88 & 8.43 & $1.75 \mathrm{e}-02$ & 2.88 \\
25 & 8513 & 9.6397 & 9.6449 & 9.6019 & 9.6440 & 5.77 & 8.31 & $4.37 \mathrm{e}-03$ & 2.75 \\
30 & 24925 & 9.6397 & 9.6415 & 9.6266 & 9.6412 & 5.73 & 8.26 & $1.51 \mathrm{e}-03$ & 2.51 \\
\hline
\end{tabular}

Table 5: [L-shaped domain, adaptive mesh refinement] Lower and upper bounds on the first exact eigenvalue $\lambda_{1}$, the effectivity indices, and the size of the relative $\lambda_{1}$ confidence interval; $\underline{\lambda}_{1}=\pi^{2} / 2$ and $\underline{\lambda}_{2}=15.1753$, Case B

\subsection{Inexact algebraic eigenvalue solvers}

We finally consider inexact eigenvalue solvers. Since we are using FreeFem ++ , we rely on an algebraic eigenvalue solver based on the ARPACK package that is built in FreeFem ++ . Here a user-specified tolerance can be provided and we choose it in a mesh-dependent way as tol $(h)=h^{2}$ to materialize an inexact solver. We set $\boldsymbol{\sigma}_{i h \text {,dis }}$ following Definition 4.1. In order to compute $\boldsymbol{\sigma}_{i h \text {,alg }}$ in (4.5), we proceed as in [47] and the references therein and first compute a second reconstructed flux $\hat{\boldsymbol{\sigma}}_{i h \text {,dis }}$ corresponding to some additional algebraic iterations (here corresponding to the tolerance $h^{2} / 100 \mathrm{in} \mathrm{ARPACK}$ ); then $\boldsymbol{\sigma}_{i h, \text { alg }}:=\hat{\boldsymbol{\sigma}}_{i h, \mathrm{dis}}-\boldsymbol{\sigma}_{i h, \text { dis }}$. Figure 6 demonstrates that we still obtain excellent lower and upper bounds. Adaptive stopping criteria of the form $(4.9 \mathrm{~b})$, leading to savings in algebraic solver iterations, are not investigated here.

\subsection{Comparison with existing results}

We finally compare our results with some existing ones from [16, 41, 39]. In what concerns the unit square and the first eigenvalue of Section 7.1, our estimates appear sharper while comparing Table 2 with the estimates presented in [16], see Figure 6.2 therein. For the L-shaped domain and uniformly refined meshes of Section 7.4 for the first eigenvalue, we also obtain better results than those presented in [16, Figure 6.4], where an efficiency issue appears; compared to the results presented in [41, Table 5.5], we observe that our lower bound $\lambda_{1 h}-\eta_{1}^{2}$ of the exact eigenvalue is a little less sharp, whereas the upper bound $\lambda_{1 h}-\tilde{\eta}_{1}^{2}$ is not present in [41]. Recall also from Section 1 that our estimates are much cheaper here than those of [41] (there is no auxiliary eigenvalue problem to solve). For adaptive meshes, we observe that our efficiency of the confidence interval for the first eigenvalue as measured in [16] by $\frac{1}{2}\left(\eta_{1}^{2}-\tilde{\eta}_{1}^{2}\right) /\left|\lambda_{1}-\lambda_{1 h}+\frac{1}{2}\left(\tilde{\eta}_{1}^{2}+\eta_{1}^{2}\right)\right|$ is approaching 1.086 which is much better than in [16, Figure 6.5].

To facilitate the comparisons, we finally present in Tables 9 and 10 several methods for the tests of [41, 

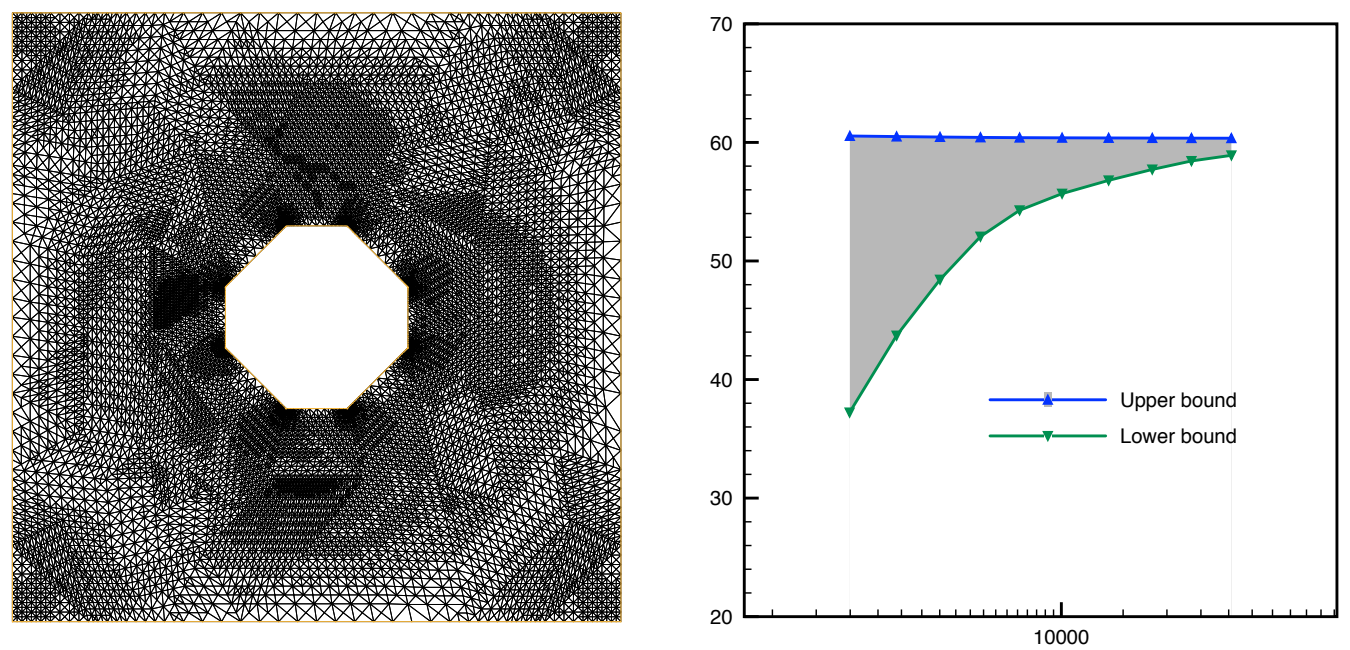

Figure 3: [Domain with a hole, adaptive mesh refinement] Mesh of the adaptive algorithm at iteration 20 (left) and the lower and upper bounds for the exact eigenvalue $\lambda_{1}$ (right); Case B

\begin{tabular}{crrrrrrrrrr}
\hline ndof & 2494 & 3390 & 4508 & 5879 & 7602 & 10047 & 13640 & 18163 & 23494 & 30533 \\
\hline$\lambda_{1 h}-\tilde{\eta}_{1}^{2}$ & 60.541 & 60.494 & 60.455 & 60.422 & 60.401 & 60.387 & 60.376 & 60.367 & 60.359 & 60.354 \\
$\lambda_{1 h}-\eta_{1}^{2}$ & 37.223 & 43.710 & 48.428 & 52.058 & 54.275 & 55.680 & 56.799 & 57.719 & 58.436 & 58.910 \\
\hline
\end{tabular}

Table 6: [Domain with a hole, adaptive mesh refinement] Lower and upper bounds on the exact eigenvalue $\lambda_{1}$ as a function of the degrees of freedom; Case B

Table $5.2(h=1 / 64)$ and Table $5.3(h=1 / 32)]$. We compare in particular the approach presented in this article, lowest-order conforming finite elements from [41], and the lowest-order Crouzeix-Raviart (CR) method presented in [16], with explicit upper bound of the interpolation constants derived in either [14] or [39]. For the eigenvalue upper bounds in the CR case, we evaluate the Rayleigh quotient on the $\mathbb{P}_{1}$ conforming nodal averaging of the original eigenvectors.

On the convex triangle, the present approach seems to give the sharpest results, whereas on the L-shaped domain, the method based on the CR finite elements with the constant from [39] is better for the lower bound. Recall, though, that important advantages of the present theory are that it additionally gives a guaranteed control of the eigenvector error by the same estimators, is not specific to a particular scheme but yields general results that are here applied to any order conforming finite element method and extended in [12] to basically any numerical scheme, and achieves polynomial-degree robustness. It can also be noted that the present estimators take elementwise form immediately suitable for adaptive mesh refinement.

\section{References}

[1] M. G. Armentano And R. G. Durán, Asymptotic lower bounds for eigenvalues by nonconforming finite element methods, Electron. Trans. Numer. Anal., 17 (2004), pp. 93-101.

[2] I. BabušKa And J. Osborn, Eigenvalue problems, in Handbook of numerical analysis, Vol. II, Handb. Numer. Anal., II, North-Holland, Amsterdam, 1991, pp. 641-787.

[3] I. BabušKa And T. Strouboulis, The finite element method and its reliability, Numerical Mathematics and Scientific Computation, The Clarendon Press Oxford University Press, New York, 2001.

[4] N. W. Bazley AND D. W. Fox, Lower bounds for eigenvalues of Schrödinger's equation, Phys. Rev. (2), 124 (1961), pp. 483-492.

[5] H. Behnke, U. Mertins, M. Plum, and C. Wieners, Eigenvalue inclusions via domain decomposition, Proc. R. Soc. Lond. A, 456 (2000), pp. 2717-2730. 

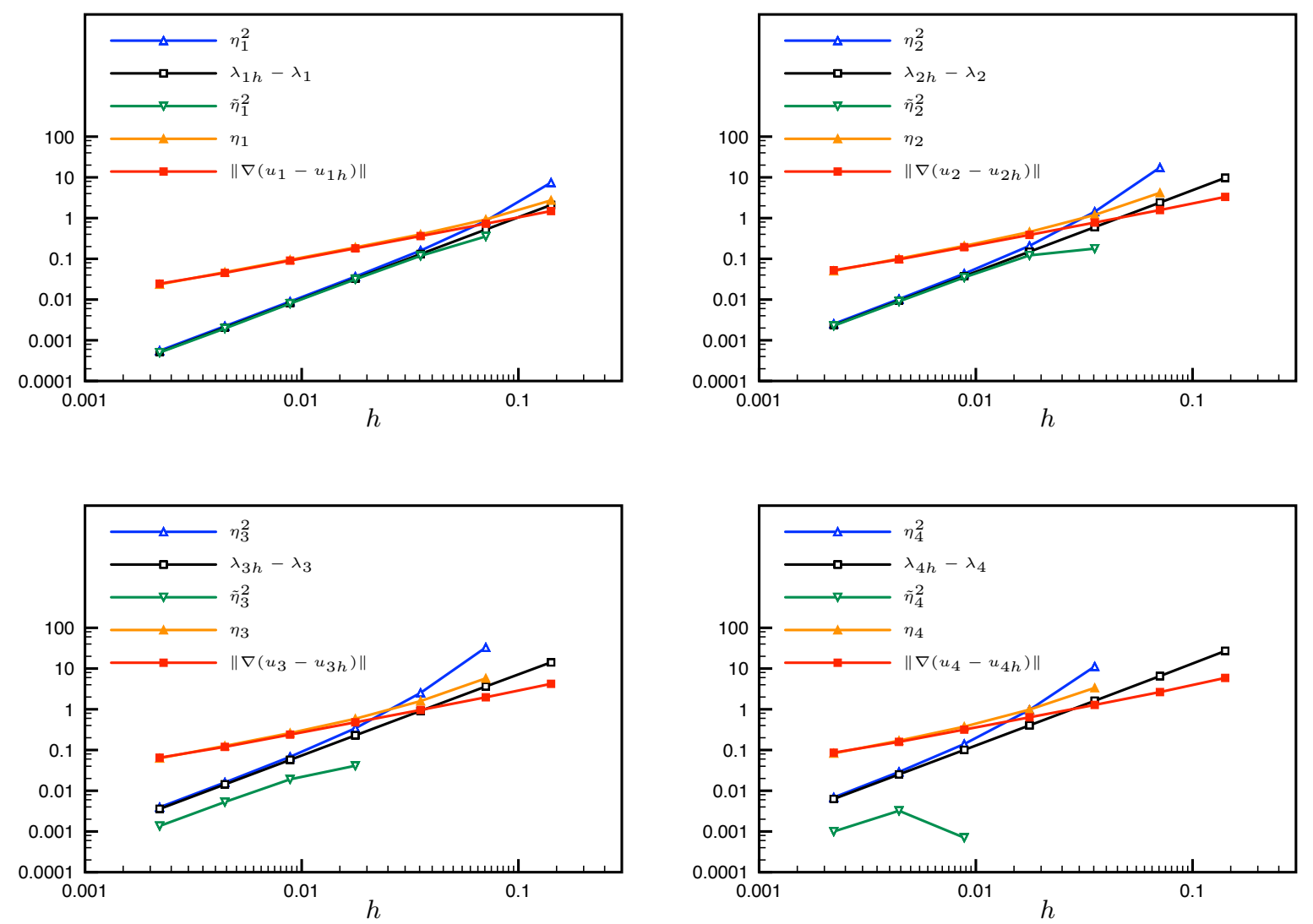

Figure 4: [Triangular domain, structured meshes] Errors in the first four eigenvalue and eigenvector approximations, their lower bounds (eigenvalues only), and their upper bounds

[6] H. Blum And M. Dobrowolski, On finite element methods for elliptic equations on domains with corners, Computing, 28 (1982), pp. 53-63.

[7] D. Boffi, Finite element approximation of eigenvalue problems, Acta Numer., 19 (2010), pp. 1-120.

[8] D. Braess, V. Pillwein, And J. SchöBerl, Equilibrated residual error estimates are p-robust, Comput. Methods Appl. Mech. Engrg., 198 (2009), pp. 1189-1197.

[9] S. C. Brenner And L. R. Scott, The mathematical theory of finite element methods, vol. 15 of Texts in Applied Mathematics, Springer, New York, third ed., 2008.

[10] F. Brezzi And M. Fortin, Mixed and hybrid finite element methods, vol. 15 of Springer Series in Computational Mathematics, Springer-Verlag, New York, 1991.

[11] E. CAncès, G. Dusson, Y. Maday, B. Stamm, and M. VohralíK, A perturbation-method-based a posteriori estimator for the planewave discretization of nonlinear Schrödinger equations, C. R. Math. Acad. Sci. Paris, 352 (2014), pp. 941-946.

[12] _ Guaranteed and robust a posteriori bounds for Laplace eigenvalues and eigenvectors: a unified framework. In preparation, 2016. 


\begin{tabular}{rcrrrrrrrrr}
\hline \multicolumn{1}{c}{$N$} & \multicolumn{1}{c}{$h$} & \multicolumn{1}{c}{ ndof } & \multicolumn{1}{c}{$\lambda_{i}$} & \multicolumn{1}{c}{$\lambda_{i h}$} & $\lambda_{i h}-\eta_{i}^{2}$ & $\lambda_{i h}-\tilde{\eta}_{i}^{2}$ & $I_{\lambda, \text { eff }}^{\text {lb }}$ & $I_{\lambda, \text { eff }}^{\text {ub }}$ & $E_{\lambda, \text { rel }}$ & $I_{u, \text { eff }}^{\text {ub }}$ \\
\hline 40 & 0.0354 & 861 & 49.3480 & 49.4789 & 49.3197 & 49.3607 & 1.11 & 1.22 & $8.29 \mathrm{e}-04$ & 1.10 \\
80 & 0.0177 & 3321 & 49.3480 & 49.3807 & 49.3442 & 49.3493 & 1.04 & 1.12 & $1.03 \mathrm{e}-04$ & 1.06 \\
160 & 0.0088 & 13041 & 49.3480 & 49.3562 & 49.3473 & 49.3482 & 1.03 & 1.09 & $1.94 \mathrm{e}-05$ & 1.05 \\
320 & 0.0044 & 51681 & 49.3480 & 49.3501 & 49.3478 & 49.3481 & 1.05 & 1.08 & $5.49 \mathrm{e}-06$ & 1.04 \\
640 & 0.0022 & 205761 & 49.3480 & 49.3485 & 49.3480 & 49.3480 & 1.02 & 1.08 & $1.07 \mathrm{e}-06$ & 1.02 \\
\hline 40 & 0.0354 & 861 & 98.6960 & 99.2953 & 97.8659 & 99.1171 & 3.36 & 2.39 & $1.27 \mathrm{e}-02$ & 1.54 \\
80 & 0.0177 & 3321 & 98.6960 & 98.8457 & 98.6376 & 98.7242 & 1.23 & 1.39 & $8.77 \mathrm{e}-04$ & 1.18 \\
160 & 0.0088 & 13041 & 98.6960 & 98.7335 & 98.6903 & 98.6985 & 1.07 & 1.15 & $8.29 \mathrm{e}-05$ & 1.08 \\
320 & 0.0044 & 51681 & 98.6960 & 98.7054 & 98.6952 & 98.6964 & 1.04 & 1.10 & $1.29 \mathrm{e}-05$ & 1.05 \\
640 & 0.0022 & 205761 & 98.6960 & 98.6984 & 98.6959 & 98.6961 & 1.03 & 1.08 & $2.54 \mathrm{e}-06$ & 1.02 \\
\hline 40 & 0.0354 & 861 & 128.3049 & 129.2175 & 126.6899 & 129.2175 & - & 2.77 & $2.30 \mathrm{e}-02$ & 1.65 \\
80 & 0.0177 & 3321 & 128.3049 & 128.5334 & 128.1923 & 128.4923 & 5.56 & 1.49 & $2.34 \mathrm{e}-03$ & 1.22 \\
160 & 0.0088 & 13041 & 128.3049 & 128.3620 & 128.2940 & 128.3429 & 3.00 & 1.19 & $3.81 \mathrm{e}-04$ & 1.09 \\
320 & 0.0044 & 51681 & 128.3049 & 128.3191 & 128.3032 & 128.3139 & 2.70 & 1.12 & $8.30 \mathrm{e}-05$ & 1.06 \\
640 & 0.0022 & 205761 & 128.3049 & 128.3084 & 128.3045 & 128.3071 & 2.62 & 1.10 & $1.99 \mathrm{e}-05$ & 1.03 \\
\hline 40 & 0.0354 & 861 & 167.7833 & 169.3980 & 158.1506 & 169.3980 & - & 6.97 & $9.48 \mathrm{e}-02$ & 2.62 \\
80 & 0.0177 & 3321 & 167.7833 & 168.1858 & 167.2205 & 168.1858 & - & 2.40 & $6.94 \mathrm{e}-03$ & 1.55 \\
160 & 0.0088 & 13041 & 167.7833 & 167.8838 & 167.7437 & 167.8831 & 142.86 & 1.39 & $8.31 \mathrm{e}-04$ & 1.18 \\
320 & 0.0044 & 51681 & 167.7833 & 167.8084 & 167.7795 & 167.8052 & 7.80 & 1.15 & $1.53 \mathrm{e}-04$ & 1.07 \\
640 & 0.0022 & 205761 & 167.7833 & 167.7896 & 167.7827 & 167.7886 & 6.29 & 1.09 & $3.49 \mathrm{e}-05$ & 1.02 \\
\hline
\end{tabular}

Table 7: [Triangular domain, uniform mesh refinement] Lower and upper bounds on the first four exact eigenvalues $\lambda_{i}$, the effectivity indices, and the sizes of the relative $\lambda_{i}$ confidence intervals

\begin{tabular}{rcrrrrrrrrr}
\hline \multicolumn{1}{c}{$N$} & \multicolumn{1}{c}{$h$} & \multicolumn{1}{c}{ ndof } & \multicolumn{1}{c}{$\lambda_{i}$} & \multicolumn{1}{c}{$\lambda_{i h}$} & $\lambda_{i h}-\eta_{i}^{2}$ & $\lambda_{i h}-\tilde{\eta}_{i}^{2}$ & $I_{\lambda, \text { eff }}^{\text {lb }}$ & $I_{\lambda, \text { eff }}^{\text {ub }}$ & $E_{\lambda, \text { rel }}$ & $I_{u, \text { eff }}^{\text {ub }}$ \\
\hline 40 & 0.0839 & 1437 & 9.6397 & 9.6955 & 9.1450 & 9.6870 & 6.59 & 9.87 & $5.76 \mathrm{e}-02$ & 3.16 \\
80 & 0.0459 & 5674 & 9.6397 & 9.6588 & 9.4719 & 9.6559 & 6.52 & 9.78 & $1.92 \mathrm{e}-02$ & 3.15 \\
160 & 0.0234 & 21878 & 9.6397 & 9.6467 & 9.5779 & 9.6456 & 6.58 & 9.86 & $7.04 \mathrm{e}-03$ & 3.16 \\
320 & 0.0125 & 86810 & 9.6397 & 9.6423 & 9.6167 & 9.6419 & 6.63 & 9.93 & $2.62 \mathrm{e}-03$ & 3.14 \\
640 & 0.0059 & 352256 & 9.6397 & 9.6407 & 9.6310 & 9.6406 & 6.73 & 9.98 & $9.94 \mathrm{e}-04$ & 2.77 \\
\hline 40 & 0.0839 & 1437 & 15.1973 & 15.2440 & 14.2080 & 15.2440 & - & 22.17 & $1.64 \mathrm{e}-01$ & 4.70 \\
80 & 0.0459 & 5674 & 15.1973 & 15.2092 & 14.9577 & 15.2092 & - & 21.11 & $4.09 \mathrm{e}-02$ & 4.60 \\
160 & 0.0234 & 21878 & 15.1973 & 15.2002 & 15.1378 & 15.2002 & - & 20.87 & $1.02 \mathrm{e}-02$ & 4.57 \\
320 & 0.0125 & 86810 & 15.1973 & 15.1980 & 15.1825 & 15.1980 & - & 20.81 & $2.55 \mathrm{e}-03$ & 4.55 \\
640 & 0.0059 & 352256 & 15.1973 & 15.1974 & 15.1936 & 15.1974 & - & 20.81 & $6.36 \mathrm{e}-04$ & 4.09 \\
\hline 40 & 0.0839 & 1437 & 19.7392 & 19.8216 & 18.7524 & 19.8216 & - & 12.97 & $1.75 \mathrm{e}-01$ & 3.59 \\
80 & 0.0459 & 5674 & 19.7392 & 19.7597 & 19.5056 & 19.7597 & - & 12.38 & $4.44 \mathrm{e}-02$ & 3.52 \\
160 & 0.0234 & 21878 & 19.7392 & 19.7444 & 19.6805 & 19.7444 & - & 12.23 & $1.14 \mathrm{e}-02$ & 3.50 \\
320 & 0.0125 & 86810 & 19.7392 & 19.7405 & 19.7246 & 19.7405 & - & 12.19 & $2.84 \mathrm{e}-03$ & 3.48 \\
640 & 0.0059 & 352256 & 19.7392 & 19.7395 & 19.7356 & 19.7395 & - & 12.20 & $7.01 \mathrm{e}-04$ & 3.07 \\
\hline 40 & 0.0839 & 1437 & 29.5215 & 29.7057 & -154.6818 & 29.7057 & - & 1000.68 & & - \\
80 & 0.0459 & 5674 & 29.5215 & 29.5675 & -10.5379 & 29.5675 & - & 871.81 & $3.08 \mathrm{e}+00$ & 29.51 \\
160 & 0.0234 & 21878 & 29.5215 & 29.5331 & 26.5255 & 29.5331 & - & 258.67 & $2.59 \mathrm{e}-01$ & 16.08 \\
320 & 0.0125 & 86810 & 29.5215 & 29.5244 & 28.8467 & 29.5244 & - & 231.37 & $6.37 \mathrm{e}-02$ & 15.16 \\
640 & 0.0059 & 352256 & 29.5215 & 29.5222 & 29.3595 & 29.5222 & - & 225.32 & $1.56 \mathrm{e}-02$ & 13.45 \\
\hline
\end{tabular}

Table 8: [L-shaped domain, uniform mesh refinement] Lower and upper bounds on the first four exact eigenvalues $\lambda_{i}$, the effectivity indices, and the sizes of the relative $\lambda_{i}$ confidence intervals

[13] C. Carstensen and S. A. Funken, Fully reliable localized error control in the FEM, SIAM J. Sci. Comput., 21 (1999/00), pp. 1465-1484.

[14] C. Carstensen and D. Gallistl, Guaranteed lower eigenvalue bounds for the biharmonic equation, Numer. Math., 126 (2014), pp. 33-51.

[15] C. Carstensen and J. Gedicke, An oscillation-free adaptive FEM for symmetric eigenvalue problems, Numer. Math., 118 (2011), pp. 401-427.

[16] — Guaranteed lower bounds for eigenvalues, Math. Comp., 83 (2014), pp. 2605-2629.

[17] C. Carstensen, J. Gedicke, and D. Rim, Explicit error estimates for Courant, Crouzeix-Raviart and Raviart-Thomas finite element methods, J. Comput. Math., 30 (2012), pp. 337-353. 

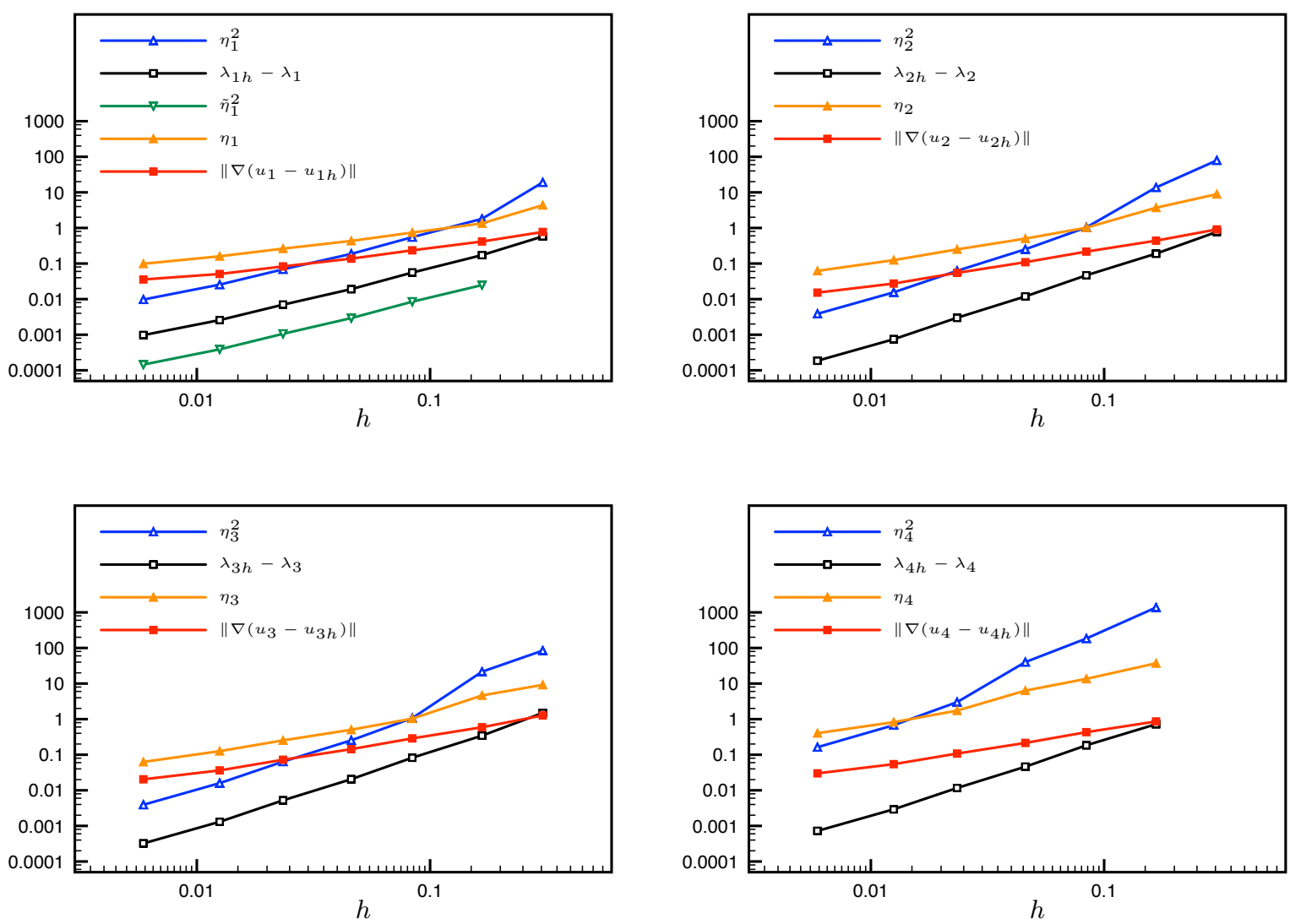

Figure 5: [L-shaped domain, unstructured meshes] Error in the first four eigenvalue and eigenvector approximations, their lower bounds (eigenvalues only), and their upper bounds

\begin{tabular}{lllll}
\hline$\lambda_{1}=49.348$ & in this work & Liu\&Oishi [41] & CR with [39] & CR with [14] \\
\hline Lower bound: & 49.341 & 49.254 & 49.288 & 49.225 \\
Upper bound: & 49.351 & 49.400 & 49.402 & \\
\hline & & & & \\
\hline$\lambda_{2}=98.696$ & in this work & Liu\&Oishi [41] & CR with [39] & CR with [14] \\
\hline Lower bound: & 98.562 & 98.352 & 98.430 & 98.179 \\
Upper bound: & 98.762 & 98.931 & 98.944 & \\
\hline
\end{tabular}

Table 9: [Triangular domain, structured meshes] Comparison of different methods; CR is the CrouzeixRaviart method based approach presented in [16] and the constants indicated in the reference

[18] P. Ciarlet, JR. AND M. Vohralík, Robust a posteriori error control for transmission problems with sign changing coefficients using localization of dual norms. HAL Preprint 01148476, submitted for publication, 2015.

[19] P. Destuynder and B. MÉTivet, Explicit error bounds in a conforming finite element method, Math. Comp., 68 (1999), pp. 1379-1396.

[20] R. G. Durán, C. Padra, and R. Rodríguez, A posteriori error estimates for the finite element 


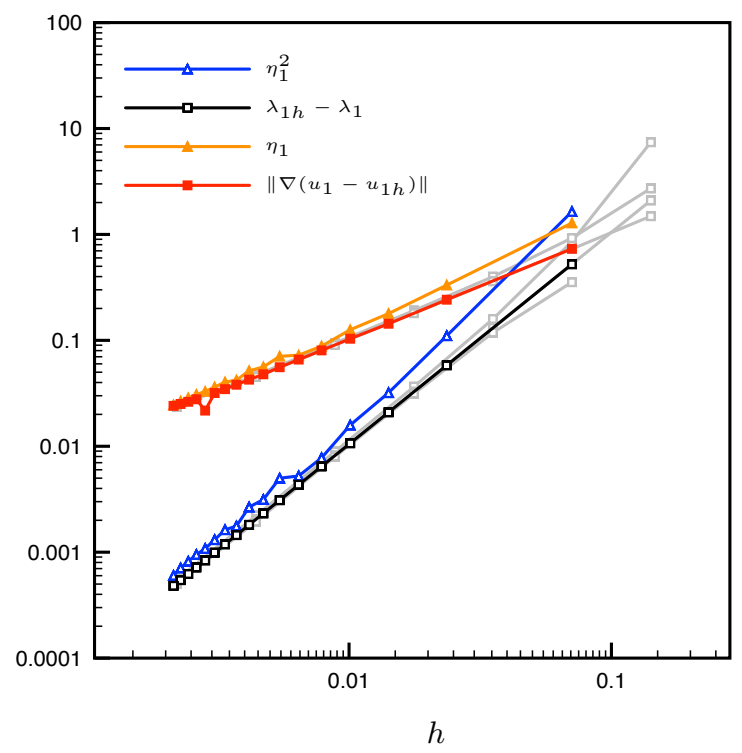

Figure 6: [Triangular domain, structured meshes, inexact solver] Error in the first eigenvalue and eigenvector approximation, its lower bound (eigenvalues only), and its upper bound for a uniform refinement; the convergence plots for an exact solver are indicated in gray

\begin{tabular}{lllll}
\hline$\lambda_{1}=9.6380$ & in this work & Liu\&Oishi [41] & CR with [39] & CR with [17] \\
\hline Lower bound: & 9.380 & 9.559 & 9.609 & 9.600 \\
Upper bound: & 9.665 & 9.670 & 9.682 & \\
\hline \multicolumn{5}{c}{} \\
\hline$\lambda_{2}=15.197$ & in this work & Liu\&Oishi [41] & CR with [39] & CR with [17] \\
\hline Lower bound: & 14.632 & 14.950 & 15.175 & 15.152 \\
Upper bound: & 15.225 & 15.225 & 15.226 & \\
\hline
\end{tabular}

Table 10: [L-shaped domain, structured meshes] Comparison of different methods; CR is the CrouzeixRaviart method based approach presented in [16] and the constants indicated in the reference

approximation of eigenvalue problems, Math. Models Methods Appl. Sci., 13 (2003), pp. 1219-1229.

[21] A. ERn and M. Vohralík, Adaptive inexact Newton methods with a posteriori stopping criteria for nonlinear diffusion PDEs, SIAM J. Sci. Comput., 35 (2013), pp. A1761-A1791.

$[22] —$ Polynomial-degree-robust a posteriori estimates in a unified setting for conforming, nonconforming, discontinuous Galerkin, and mixed discretizations, SIAM J. Numer. Anal., 53 (2015), pp. 1058 1081.

[23] _ Stable broken $H^{1}$ and $\mathbf{H}(\mathrm{div})$ polynomial extensions for polynomial-degree-robust potential and flux reconstruction in three space dimensions. In preparation, 2016.

[24] G. E. Forsythe, Asymptotic lower bounds for the fundamental frequency of convex membranes, Pacific J. Math., 5 (1955), pp. 691-702.

[25] D. W. Fox And W. C. Rheinboldt, Computational methods for determining lower bounds for eigenvalues of operators in Hilbert space, SIAM Rev., 8 (1966), pp. 427-462.

[26] D. Gilbarg and N. S. Trudinger, Elliptic partial differential equations of second order, vol. 224 of Grundlehren der Mathematischen Wissenschaften [Fundamental Principles of Mathematical Sciences], Springer-Verlag, Berlin, second ed., 1983. 
[27] F. Goerisch And Z. Q. HE, The determination of guaranteed bounds to eigenvalues with the use of variational methods. I, in Computer arithmetic and self-validating numerical methods (Basel, 1989), vol. 7 of Notes Rep. Math. Sci. Engrg., Academic Press, Boston, MA, 1990, pp. 137-153.

[28] P. Grisvard, Elliptic problems in nonsmooth domains, vol. 24 of Monographs and Studies in Mathematics, Pitman (Advanced Publishing Program), Boston, MA, 1985.

[29] L. Grubišić And J. S. Ovall, On estimators for eigenvalue/eigenvector approximations, Math. Comp., 78 (2009), pp. 739-770.

[30] F. Hecht, New development in FreeFem++, J. Numer. Math., 20 (2012), pp. 251-265.

[31] V. Heuveline And R. Rannacher, A posteriori error control for finite approximations of elliptic eigenvalue problems, Adv. Comput. Math., 15 (2001), pp. 107-138 (2002).

[32] J. Hu, Y. HuAnG, AND Q. Lin, Lower bounds for eigenvalues of elliptic operators: by nonconforming finite element methods, J. Sci. Comput., 61 (2014), pp. 196-221.

[33] J. Hu, Y. HuAng, AND Q. Shen, The lower/upper bound property of approximate eigenvalues by nonconforming finite element methods for elliptic operators, J. Sci. Comput., 58 (2014), pp. 574-591.

[34] T. Kato, On the upper and lower bounds of eigenvalues, J. Phys. Soc. Japan, 4 (1949), pp. $334-339$.

[35] J. R. Kuttler And V. G. Sigillito, Bounding eigenvalues of elliptic operators, SIAM J. Math. Anal., 9 (1978), pp. 768-778.

[36] — Estimating eigenvalues with a posteriori/a priori inequalities, vol. 135 of Research Notes in Mathematics, Pitman (Advanced Publishing Program), Boston, MA, 1985.

[37] Y. A. Kuznetsov And S. I. Repin, Guaranteed lower bounds of the smallest eigenvalues of elliptic differential operators, J. Numer. Math., 21 (2013), pp. 135-156.

[38] M. G. LARSOn, A posteriori and a priori error analysis for finite element approximations of self-adjoint elliptic eigenvalue problems, SIAM J. Numer. Anal., 38 (2000), pp. 608-625.

[39] X. LIU, A framework of verified eigenvalue bounds for self-adjoint differential operators, Appl. Math. Comput., 267 (2015), pp. 341-355.

[40] X. LiU AND F. KIKUCHI, Analysis and estimation of error constants for $P_{0}$ and $P_{1}$ interpolations over triangular finite elements, J. Math. Sci. Univ. Tokyo, 17 (2010), pp. 27-78.

[41] X. LiU AND S. OISHI, Verified eigenvalue evaluation for the Laplacian over polygonal domains of arbitrary shape, SIAM J. Numer. Anal., 51 (2013), pp. 1634-1654.

[42] F. LuO, Q. LIN, AND H. XIE, Computing the lower and upper bounds of Laplace eigenvalue problem: by combining conforming and nonconforming finite element methods, Sci. China Math., 55 (2012), pp. 1069-1082.

[43] Y. MAdAy And A. T. PATERA, Numerical analysis of a posteriori finite element bounds for linear functional outputs, Math. Models Methods Appl. Sci., 10 (2000), pp. 785-799.

[44] V. Mehrmann and A. Miedlar, Adaptive computation of smallest eigenvalues of self-adjoint elliptic partial differential equations, Numer. Linear Algebra Appl., 18 (2011), pp. 387-409.

[45] C. B. Moler AND L. E. PAYNe, Bounds for eigenvalues and eigenvectors of symmetric operators, SIAM J. Numer. Anal., 5 (1968), pp. 64-70.

[46] J. PApež, U. RÜDE, M. Vohralík, And B. Wohlmuth, Sharp algebraic and total a posteriori error bounds via a multilevel approach. In preparation, 2016.

[47] J. PApež, Z. Strakoš, And M. Vohralík, Estimating and localizing the algebraic and total numerical errors using flux reconstructions. HAL Preprint 01312430, submitted for publication, 2016. 
[48] M. Plum, Guaranteed numerical bounds for eigenvalues, in Spectral theory and computational methods of Sturm-Liouville problems (Knoxville, TN, 1996), vol. 191 of Lecture Notes in Pure and Appl. Math., Dekker, New York, 1997, pp. 313-332.

[49] W. Prager and J. L. Synge, Approximations in elasticity based on the concept of function space, Quart. Appl. Math., 5 (1947), pp. 241-269.

[50] R. Rannacher, A. Westenberger, and W. Wollner, Adaptive finite element solution of eigenvalue problems: balancing of discretization and iteration error, J. Numer. Math., 18 (2010), pp. 303-327.

[51] S. Repin, A posteriori estimates for partial differential equations, vol. 4 of Radon Series on Computational and Applied Mathematics, Walter de Gruyter GmbH \& Co. KG, Berlin, 2008.

[52] J. E. Roberts and J.-M. Thomas, Mixed and hybrid methods, in Handbook of Numerical Analysis, Vol. II, North-Holland, Amsterdam, 1991, pp. 523-639.

[53] Y. SAAD, Numerical methods for large eigenvalue problems, Algorithms and Architectures for Advanced Scientific Computing, Manchester University Press, Manchester; Halsted Press [John Wiley \& Sons, Inc.], New York, 1992.

[54] I. ŠEBestovÁ AND T. VEJChODSKÝ, Two-sided bounds for eigenvalues of differential operators with applications to Friedrichs, Poincaré, trace, and similar constants, SIAM J. Numer. Anal., 52 (2014), pp. 308-329.

[55] — Two-sided bounds of eigenvalues - local efficiency and convergence of adaptive algorithm. ArXiv preprint 1606.01739v1, 2016.

[56] P. Solin and S. Giani, An iterative adaptive finite element method for elliptic eigenvalue problems, J. Comput. Appl. Math., 236 (2012), pp. 4582-4599.

[57] G. Still, Computable bounds for eigenvalues and eigenfunctions of elliptic differential operators, $\mathrm{Nu-}$ mer. Math., 54 (1988), pp. 201-223.

[58] G. Strang and G. Fix, An analysis of the finite element method, Wellesley-Cambridge Press, Wellesley, MA, second ed., 2008.

[59] L. N. Trefethen And T. Betcke, Computed eigenmodes of planar regions, in Recent advances in differential equations and mathematical physics, vol. 412 of Contemp. Math., Amer. Math. Soc., Providence, RI, 2006, pp. 297-314.

[60] R. Verfürth, A posteriori error estimates for nonlinear problems. Finite element discretizations of elliptic equations, Math. Comp., 62 (1994), pp. 445-475.

[61] H. F. Weinberger, Upper and lower bounds for eigenvalues by finite difference methods, Comm. Pure Appl. Math., 9 (1956), pp. 613-623.

[62] H. Xie, M. Yue, And N. Zhang, Fully computable error bounds for eigenvalue problem. ArXiv Preprint 1601.01561v1, 2016.

[63] Y. Yang, J. Han, H. Bi, AND Y. YU, The lower/upper bound property of the Crouzeix-Raviart element eigenvalues on adaptive meshes, J. Sci. Comput., 62 (2015), pp. 284-299. 